\title{
A Tool for Integer Homology Computation: $\lambda$-AT-Model
}

\author{
R. Gonzalez-Diaz,, M.J. Jimenez,, B. Medrano, , P. Real \\ Applied Math Department, University of Sevilla, \\ Campus Reina Merdedes, CP: 41012, Sevilla, Spain \\ \{rogodi,majiro,belenmg,real\}@us.es \\ http://alojamientos.us.es/gtocoma
}

\begin{abstract}
In this paper, we formalize the notion of $\lambda$-AT-model (where $\lambda$ is a nonnull integer) for a given chain complex, which allows the computation of homological information in the integer domain avoiding using the Smith Normal Form of the boundary matrices. We present an algorithm for computing such a model, obtaining Betti numbers, the prime numbers $p$ involved in the invariant factors of the torsion subgroup of homology, the amount of invariant factors that are a power of $p$ and a set of representative cycles of generators of homology $\bmod p$, for each $p$. Moreover, we establish the minimum valid $\lambda$ for such a construction, what cuts down the computational costs related to the torsion subgroup. The tools described here are useful to determine topological information of $\mathrm{nD}$ structured objects such as simplicial, cubical or simploidal complexes and are applicable to extract such an information from digital pictures.
\end{abstract}

Keywords: algebraic topological model, $\mathrm{nD}$ digital image, integer homology, chain complex

\section{Introduction}

Important questions in Vision and Image Processing involve an accurate topological analysis. In some scientific areas, such as Medical Imaging and Remote Sensing, there is a growing need to visualize, analyze and manipulate data of high complexity and dimensionality. We are concerned about providing algorithms to solve topological problems in any dimension. For 
this aim, efficient methods from combinatorial or algebraic topology to compute topological features are needed. Due to the fact that we will work with n-dimensional combinatorial objects (mainly, $n=3,4 \ldots$ ), topological features are not limited to Betti numbers, Euler characteristic, connectivity, number of holes or cavities, but also include other advanced characteristics such as (co)homology in the integer domain, cohomology ring, cohomology operations or homotopy groups, which can help to discriminate topologically non-equivalent objects. Algebraic Topology and Homological Algebra fields provide the necessary tools to capture all this topological stuff (see [14, 12, 17]).

The classical algorithm for computing homology groups (in the integer domain) uses the Smith Normal Form (SNF) of the boundary matrices of a chain complex. Explicit examples can be given for which the computation of SNF has a worst-case computational complexity which grows exponentially in both space and time [3]. Many algorithms have been devised to improve this complexity bound [11, 18, 2, 15].

In the AT-model theory [5, 6], starting from a simplicial complex $K$, a chain homotopy equivalence is generated from the chain complex associated to $K, \mathcal{C}(K)$, to its homology. In this case, the complexity of the associated algorithm is $\mathcal{O}\left(\mathrm{m}^{3}\right)$, where $m$ is the number of generators of the complex, but the computation is carried out over a field. In this paper, we extend the notion of AT-model for integer homology computation. More concretely, we define the homological algebra notion of $\lambda$-AT-model and describe an algorithm for computing a structure of this kind from any chain complex, with coefficients in the integer domain $(\mathbf{Z})$. Starting from this information, it is possible, in particular, to obtain Betti numbers, the prime numbers $p$ involved in the invariant factors (corresponding to the torsion subgroup of the homology), the amount of invariant factors that are a power of $p$ and a set of representative cycles of generators of homology $\bmod p$, without using SNF. We present an algorithm for computing such a $\lambda$-AT-model for a given chain complex and extracting all this homological information, performed in $\mathcal{O}\left(m^{3} \psi(\lambda)\right)$ in the worst case, $\psi$ being the Euler function.

In the following section, we recall classical definitions from Algebraic Topology. In Section 3, previous tools for computing topological information such as AM-models (which make use of SNF), and AT-models (for computing homological information over a field) are given. In Section 4, we define the notion of $\lambda$-AT-model, study its properties, give an algorithm for computing it and study its complexity. Finally, we describe how to extract homological 
information in the integer domain from $\lambda$-AT-models. The last section is devoted to conclusions and future work.

\section{Background}

This section introduces the basic concepts and definitions needed throughout the paper, taking as main reference Munkres' book [14].

A chain complex $\mathcal{C}$ is a sequence $\ldots \stackrel{d_{q+1}}{\rightarrow} \mathcal{C}_{q} \stackrel{d_{q}}{\rightarrow} \mathcal{C}_{q-1} \stackrel{d_{q-1}}{\rightarrow} \ldots \stackrel{d_{1}}{\rightarrow} \mathcal{C}_{0} \stackrel{d_{0}}{\rightarrow} 0$ of free abelian groups $\mathcal{C}_{q}$ and homomorphisms $d_{q}: \mathcal{C}_{q} \rightarrow \mathcal{C}_{q-1}$, such that, for all $q, d_{q} d_{q+1}=0$. An element $a \in \mathcal{C}_{q}$ is a $q$-chain of $\mathcal{C}$; we say that $q$ is the dimension of $a$, and write $q=\operatorname{dim}(a)$. The set $d=\left\{d_{q}\right\}$ is called the differential of $\mathcal{C}$. If there is no confusion, we can write $a \in \mathcal{C}$ if $a \in \mathcal{C}_{q}$ for some $q$. Similarly, we can omit the subindex $q$ in $d_{q}(a)$.

The chain complex $\mathcal{C}$ is finite if there exists an integer $n>0$ such that $\mathcal{C}_{q}=0$ for $q>n$ and each abelian group $\mathcal{C}_{q}$ is finitely generated. In this case, if $\mathcal{C}_{n} \neq 0$, we say that $\operatorname{dim}$ of $\mathcal{C}$ is $n$.

Since our goal is the computation of homological information of finite objects, all chain complexes considered here are finite. In this case, $\mathcal{C}$ can be encoded as a pair $(C, d)$, where: (1) $C=\left\{C_{q}\right\}$, and $C_{q}$ is a set of generators of $\mathcal{C}_{q} ;(2) d=\left\{d_{q}\right\}$ and for each $q, d_{q}$ is the differential of $\mathcal{C}$ in $\operatorname{dim} q$ with respect to the bases $C_{q}$ and $C_{q-1}$. Sometimes, we write $\mathcal{C}=(C, d)$ if the chain complex $\mathcal{C}$ is encoded as the pair $(C, d)$. If $b \in \mathcal{C}_{q}$ and $a \in C_{q}$, then $\langle b, a\rangle$ denotes the coefficient of the generator $a$ in the $q$-chain $b$ when $b$ is expressed as a linear combination of elements of $C_{q}$.

Example 2.1. Shapes are classically modeled with cellular subdivisions. Several combinatorial structures may represent such a subdivision. Simplicial complexes have proved to be a useful tool to model a geometric object. Roughly speaking, they are collections of simplices (vertices, edges, triangles, tetrahe$d r a, \ldots)$ that fit together in a natural way to form the object. A q-simplex $\sigma$ is a convex hull of a set of $q$ affinely independent points. If the set is $\left\{v_{0}, v_{1}, \ldots, v_{q}\right\}$ then the simplex is denoted by $v_{0} v_{1} \cdots v_{q}$. The boundary operator over the $q$-simplex $\sigma$ is defined by $\partial_{q}\left(v_{0} v_{1} \cdots v_{q}\right)=\sum_{i=0}^{q}(-1)^{i} v_{0} v_{1} \cdots \hat{v}_{i} \cdots v_{q}$ where $\hat{v}_{i}$ means that the vertex $v_{i}$ is omitted. For every simplicial complex $K$, one can define a chain complex $\mathcal{C}(K)$ (canonically associated to it) such that the q-chains are linear combinations of q-simplices. By linearity, the boundary operator can be extended to chains to define the differential $\partial$ of $\mathcal{C}(K)$. The homology of $K$ is then defined as the homology of $\mathcal{C}(K)$. Another 

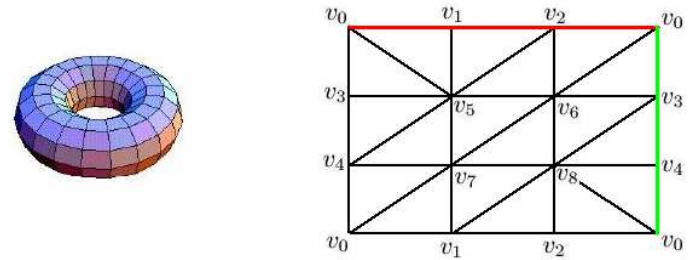

Figure 1: The Torus and a triangulation $T$ of it.
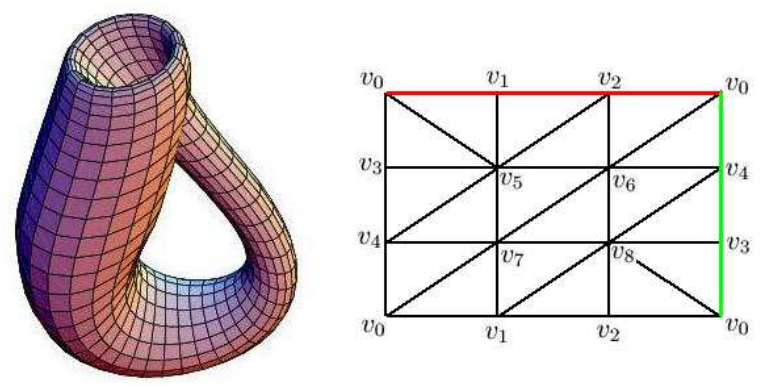

Figure 2: The Klein bottle and a triangulation $S$ of it.

way of combinatorial representation of a geometric structure (which could arise naturally, for example, from tomography, numerical computations and graphics), is by means of cubical grids, which subdivide the space into cubes with vertices in an integer lattice. This approach, that can be generalized to an arbitrary dimension, leads to a cubical complex. The homology of a given cubical complex is the homology of the cubical chain complex associated to it [13]. Finally, simploidal sets [1] include simplicial complexes and cubical complexes as particular cases. They can be used for representing 'hybrid' grids coming from finite element methods. In [16], a free chain complex is associated to a simploidal set and the homology of the simploidal set is defined as the homology of the associated chain complex.

Given a chain complex $\mathcal{C}$ with differential $d$, a $q$-chain $a \in \mathcal{C}_{q}$ is called a $q$-cycle if $d_{q}(a)=0$. If $a=d_{q+1}(b)$ for some $b \in \mathcal{C}_{q+1}$ then $a$ is called a $q$-boundary. Denote the groups of $q$-cycles and $q$-boundaries by $Z_{q}$ and $B_{q}$ respectively. We say that two $q$-cycles $a$ and $b$ are homologous if there exists 
a $(q+1)$-chain $c$ such that $a=b+d_{q+1}(c)$. Define the integer $q$ th homology group to be the quotient group $Z_{q} / B_{q}$, denoted by $H_{q}(\mathcal{C} ; \mathbf{Z})$.

For each $q$, the integer $q$ th homology group $H_{q}(\mathcal{C} ; \mathbf{Z})$ is a finitely generated abelian group. Moreover, $H_{q}(\mathcal{C} ; \mathbf{Z})$ is isomorphic to $F_{q}(\mathcal{C} ; \mathbf{Z}) \oplus T_{q}(\mathcal{C} ; \mathbf{Z})$ where

$$
F_{q}(\mathcal{C} ; \mathbf{Z})=\mathbf{Z} \oplus \cdots \oplus \mathbf{Z} \quad \text { and } \quad T_{q}(\mathcal{C} ; \mathbf{Z})=\left(\mathbf{Z} / \alpha_{(q, 1)}\right) \oplus \cdots \oplus\left(\mathbf{Z} / \alpha_{(q, s)}\right)
$$

are the free subgroup and the torsion subgroup of $H_{q}(\mathcal{C} ; \mathbf{Z})$, respectively. The rank of $F_{q}(\mathcal{C} ; \mathbf{Z})$, denoted by $\beta_{q}$, is called the $q$ th Betti number of $\mathcal{C}$. Intuitively, $\beta_{0}$ is the number of connected components, $\beta_{1}$ is the number of independent holes and $\beta_{2}$ is the number of cavities. Each $\alpha_{(q, i)}$ is a power of a prime, $\alpha_{(q, i)}=p_{i}^{t_{\left(q, p_{i}\right)}}$. They are called the invariant factors of $H_{q}(\mathcal{C} ; \mathbf{Z})$. The numbers $\beta_{q}$ and $\alpha_{(q, i)}$ are uniquely determined by $H_{q}(\mathcal{C} ; \mathbf{Z}$ ) (up to a rearrangement). Therefore, this representation is in some sense a 'canonical form' for $H_{q}(\mathcal{C} ; \mathbf{Z})$. For all $q$, there exists a finite number of elements of $H_{q}(\mathcal{C} ; \mathbf{Z})$ from which we can deduce all the $H_{q}(\mathcal{C} ; \mathbf{Z})$ elements. Those elements are called homology generators of $\operatorname{dim} q$. We say that $a$ is a representative $q$-cycle of a homology generator $\alpha$ of the free subgroup of $H_{q}(\mathcal{C} ; \mathbf{Z})$ if $\alpha=a+B_{q}$ and for each $\mu \in \mathbf{Z}, \mu \neq 0, \mu a \notin \operatorname{Im} d$. We say that $a$ is a representative $q$-cycle of a homology generator $\alpha$ of the torsion subgroup of $H_{q}(\mathcal{C} ; \mathbf{Z})$ if $\alpha=a+B_{q}$ and there exists $\mu \in \mathbf{Z}, \mu \neq 0$, such that $\mu a \in \operatorname{Im} d$. We denote $\alpha=[a]$.

Proposition 2.2. [14, $p$. 332] The qth homology group of $\mathcal{C}$ with coefficients in $\mathbf{Z} / p$ for $p$ being a prime, denoted by $H_{q}(\mathcal{C} ; \mathbf{Z} / p)$, is a vector space. Its rank, denoted by $\beta_{(q, p)}$, depends on the prime $p$ and it is related to the number of invariant factors of $H_{q}(\mathcal{C} ; \mathbf{Z})$ that are a power of $p, T_{(q, p)}$, via the Universal Coefficient Theorem for Homology, which implies that, for each prime $p$,

$$
T_{(q, p)}=\beta_{(q, p)}-\beta_{q}-T_{(q-1, p)} \text { for } q>0 \text { and } T_{(0, p)}=\beta_{(0, p)}-\beta_{0} .
$$

Example 2.3. Consider a triangulation $T$ of the torus and a triangulation $S$ of the Klein bottle (see Fig. 1). Let $\mathcal{C}(T)$ and $\mathcal{C}(S)$ be the chain complexes canonically associated to $T$ and $S$, respectively.

The homology groups of the torus are:

$$
H_{0}(\mathcal{C}(T) ; \mathbf{Z}) \simeq \mathbf{Z}, H_{1}(\mathcal{C}(T) ; \mathbf{Z}) \simeq \mathbf{Z} \oplus \mathbf{Z}, H_{2}(\mathcal{C}(T) ; \mathbf{Z}) \simeq \mathbf{Z}
$$

The Betti numbers for the torus are: $\beta_{0}^{T}=1, \beta_{1}^{T}=2$ and $\beta_{2}^{T}=1$. Representative cycles of homology generators of dim 1 are shown in red and green 
in Fig. 1, respectively. The 2-chain $\tau$ which is the sum (up to signs) of all the triangles of $T$ satisfies that $\partial_{2}(\tau)=0$, so $\tau$ is a representative cycle of a homology generator of dim 2.

In the case of the Klein bottle, its homology groups are:

$$
H_{0}(\mathcal{C}(S) ; \mathbf{Z}) \simeq \mathbf{Z}, H_{1}(\mathcal{C}(S) ; \mathbf{Z}) \simeq \mathbf{Z} \oplus \mathbf{Z} / 2, H_{2}(\mathcal{C}(S) ; \mathbf{Z}) \simeq 0
$$

The Betti numbers of the Klein bottle are $\beta_{0}^{S}=1, \beta_{1}^{S}=1$ and $\beta_{2}^{S}=0$. A representative cycle of a homology generator of the free subgroup of $H_{1}(\mathcal{C}(S) ; \mathbf{Z})$ is shown in red in Fig包. The invariant factor of $H_{1}(\mathcal{C}(S) ; \mathbf{Z})$ is 2. The representative cycle $\alpha$ of a homology generator of the torsion subgroup of $H_{1}(\mathcal{C}(S) ; \mathbf{Z})$, which is shown in green in Figure 2, satisfies that $\partial_{2}(\gamma)=2 \alpha$ where $\gamma$ is the sum (up to signs) of all the triangles of $S$.

Working with coefficients in a field, it is not possible, in general, to get all the information corresponding to the torsion subgroup of the homology. For example, the homology groups of $S$ with coefficients in $\mathbf{Z} / 3$ are

$$
H_{0}(\mathcal{C}(S) ; \mathbf{Z} / 3)=\mathbf{Z} / 3, H_{1}(\mathcal{C}(S) ; \mathbf{Z} / 3)=\mathbf{Z} / 3, H_{2}(\mathcal{C}(S) ; \mathbf{Z} / 3)=0
$$

since $\partial_{2}(\gamma)=2 \alpha$, so $\partial_{2}(2 \gamma)=\alpha$. Notice that the torsion part is lost since the prime $p=3$ is not involved in any invariant factor of $H(\mathcal{C}(S), \mathbf{Z})$. The homology groups of the Klein bottle with coefficients in $\mathbf{Q}$ are

$$
H_{0}(\mathcal{C}(S) ; \mathbf{Q})=\mathbf{Q}, H_{1}(\mathcal{C}(S) ; \mathbf{Q})=\mathbf{Q}, H_{2}(\mathcal{C}(S) ; \mathbf{Q})=0
$$

since $\partial_{2}\left(\frac{1}{2} \gamma\right)=\alpha$. Finally, the homology groups of the Klein bottle with coefficients in $\mathbf{Z} / 2$ are

$$
H_{0}(\mathcal{C}(S) ; \mathbf{Z} / 2)=\mathbf{Z} / 2, H_{1}(\mathcal{C}(S) ; \mathbf{Z} / 2)=\mathbf{Z} / 2 \oplus \mathbf{Z} / 2, H_{2}(\mathcal{C}(S) ; \mathbf{Z} / 2)=\mathbf{Z} / 2
$$

since $\partial_{2}(\gamma)=2 \alpha=0$.

Let $\mathcal{C}$ and $\mathcal{C}^{\prime}$ be two chain complexes. Denote the differential of $\mathcal{C}$ and $\mathcal{C}^{\prime}$ by $d$ and $d^{\prime}$, respectively. A chain map $f: \mathcal{C} \rightarrow \mathcal{C}^{\prime}$ is a family of homomorphisms $\left\{f_{q}: C_{q} \rightarrow C_{q}^{\prime}\right\}$ such that $d_{q}^{\prime} f_{q}=f_{q-1} d_{q}$ for all $q \geq 0$. If no confusion can arise, we omit the subindex and write $f(a)$ instead of $f_{q}(a)$ for any $q$ and $a \in \mathcal{C}_{q}$. A chain map $f: \mathcal{C} \rightarrow \mathcal{C}^{\prime}$ induces a homomorphism $[f]: H(\mathcal{C} ; \mathbf{Z}) \rightarrow$ $H\left(\mathcal{C}^{\prime} ; \mathbf{Z}\right)$ where $[f]([a])=[f(a)]$ for every $[a] \in H(\mathcal{C} ; \mathbf{Z})$. If $f, g: \mathcal{C} \rightarrow \mathcal{C}^{\prime}$ are chain maps, then a chain homotopy $\phi: \mathcal{C} \rightarrow \mathcal{C}^{\prime}$ of $f$ to $g$ is a family of homomorphisms $\left\{\phi_{q}: C_{q} \rightarrow C_{q+1}^{\prime}\right\}$ such that $f_{q}-g_{q}=d_{q+1}^{\prime} \phi_{q}+\phi_{q-1} d_{q}$. A 


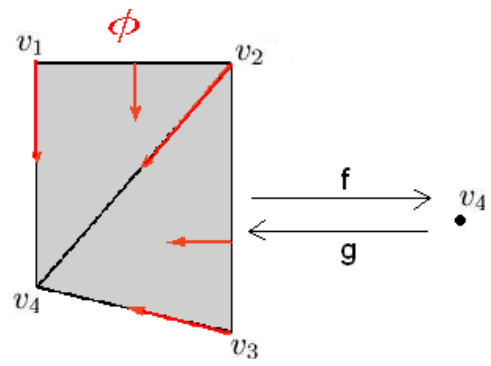

Figure 3: The chain contraction $(f, g, \phi)$ of $\mathcal{C}(K)$ to $\mathcal{C}(L)$.

chain contraction of a chain complex $\mathcal{C}$, to another chain complex $\mathcal{C}^{\prime}$ with differentials $d$ and $d^{\prime}$, respectively, is a set $(f, g, \phi)$ such that: $f: \mathcal{C} \rightarrow \mathcal{C}^{\prime}$ and $g: \mathcal{C}^{\prime} \rightarrow \mathcal{C}$ are chain maps; $f g$ is the identity map of $\mathcal{C}^{\prime}$ (denoted by $i d_{\mathcal{C}^{\prime}}$ ) and $\phi: \mathcal{C} \rightarrow \mathcal{C}$ is a chain homotopy of the identity map of $\mathcal{C}$ to $g f$, that is, $\phi d+d \phi=i d_{\mathcal{c}}-g f$. Important properties of chain contractions are: (1) $g$ is injective and $f$ is surjective since $f g=i d_{\mathcal{C}^{\prime}}$ and, in particular, $\mathcal{C}^{\prime}$ has fewer or the same number of generators than $\mathcal{C} ;(2)[f][g]=i d_{H\left(\mathcal{C}^{\prime} ; \mathbf{z}\right)}$ and $[g][f]=i d_{H(\mathcal{C} ; \mathbf{z})}$ and, therefore, the homology groups of $\mathcal{C}$ and $\mathcal{C}^{\prime}$ are isomorphic [13].

Example 2.4. Let $K$ be the simplicial complex given by the following set of simplices (see Fig. 3 on the left):

$$
\left\{v_{1}, v_{2}, v_{3}, v_{4}, v_{1} v_{2}, v_{2} v_{3}, v_{3} v_{4}, v_{2} v_{4}, v_{1} v_{4}, v_{1} v_{2} v_{4}, v_{2} v_{3} v_{4}\right\}
$$

and let $L$ be the simplicial complex given by the vertex $v_{4}$.

Let $f: \mathcal{C}(K) \rightarrow \mathcal{C}(L)$ and $g: \mathcal{C}(L) \rightarrow \mathcal{C}(K)$ be the chain maps given by $f\left(v_{i}\right)=v_{4}$ for $i=1,2,3,4$ and $f$ is null over the other simplices; $g\left(v_{4}\right)=v_{4}$. Let $\phi: \mathcal{C}(K) \rightarrow \mathcal{C}(K)$ be the chain homotopy of $i d_{\mathcal{C}(K)}$ to gf given by: $\phi\left(v_{1}\right)=$ $v_{1} v_{4}, \phi\left(v_{2}\right)=v_{2} v_{4}, \phi\left(v_{3}\right)=v_{3} v_{4}, \phi\left(v_{1} v_{2}\right)=v_{1} v_{2} v_{4}, \phi\left(v_{2} v_{3}\right)=v_{2} v_{3} v_{4}$ and $\phi$ is null over the other simplices. The set $(f, g, \phi)$ is a chain contraction of $\mathcal{C}(K)$ to $\mathcal{C}(L)$ and hence, $\mathcal{C}(K)$ and $\mathcal{C}(L)$ have isomorphic homology groups. Fig. 3 illustrates such a chain contraction. Observe that the action of the map $\phi$ 'reduces' the simplicial complex to its homology. 


\section{AM-model and AT-model}

We recall here the concepts of AM-model and AT-model. Both tools represent the prior work that lays the foundations of $\lambda$-AT-model. In fact, the latter attempts to take the qualities of each of the former, computing homological information in the integer domain avoinding the computation of SNF.

An algebraic minimal model (AM-model) [4] is a tool for computing topological information of chain complexes, tool that is applicable to extract topological information from digital pictures. In particular, it provides integer (co)homology generators, representative (co)cycles of these generators as well as the cohomological invariant $H B 1$ (derived from the rank of the cohomology ring) [4, 7, 8]. More concretely, an AM-model for a chain complex $\mathcal{C}=(C, d)$ is a set $\left((C, d),\left(M, d^{\prime}\right) f, g, \phi\right)$, where

(i) $M=\left\{M_{q}\right\}$ generates a chain complex, denoted by $\mathcal{M}$, with differential $d^{\prime}$ such that any non-null entry of SNF of the matrix of $d^{\prime}$ in each dim $q$, is greater than 1 .

(ii) $(f, g, \phi)$ defines a chain contraction of $\mathcal{C}$ to $\mathcal{M}$.

Property (ii) follows that $H(\mathcal{C} ; \mathbf{Z})$ and $H(\mathcal{M} ; \mathbf{Z})$ have isomorphic homology groups. Moreover, integer (co)homology generators and representative (co)cycles of (co)homology generators of $\mathcal{C}$ can directly be obtained from $\mathcal{M}$. See Fig. 4, for example, where $M_{1}=\{\alpha, \beta\}, \alpha$ being a representative cycle of a generator of the free part of $H_{1}(\mathcal{C}(S) ; \mathbf{Z})$ and $\beta$ a representative cycle of a generator of the torsion part. An algorithm for computing AM-models is given in [7, 8]. It needs to reduce the matrices of the differential to its SNF.

An algebraic topological model (AT-model) [5, 6] is a tool for computing over a field $\mathbf{Z} / p, p$ being a prime, (co)homology, representative (co)cycles of (co)homology generators and the cup product on cohomology of $\mathrm{nD}$ digital images (and hence also the cohomological invariant $H B 1$ ). The algorithm presented in [5, 6] for computing AT-models over fields runs in time at most $\mathcal{O}\left(\mathrm{m}^{3}\right)$, where $m$ is the number of generators of the given chain complex. More concretely, an $A T$-model for a chain complex $\mathcal{C}=(C, d)$ is a set $((C, d), H, f, g, \phi)$, where

(i) $H=\left\{H_{q}\right\}$ generates a chain complex, denoted by $\mathcal{H}$, with null differential. 


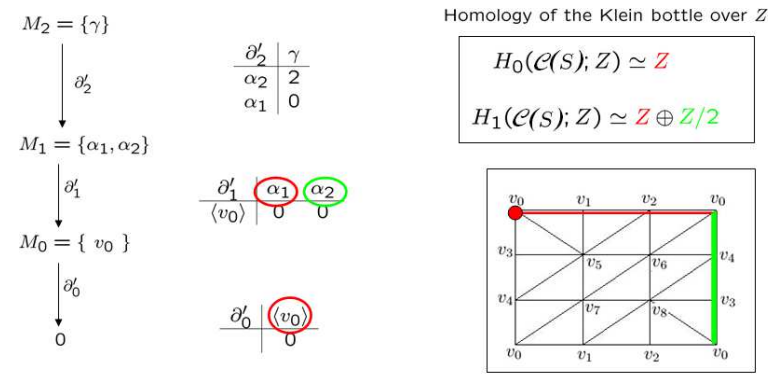

Figure 4: Homological information obtained from an AM-model for the Klein bottle.

(ii) $(f, g, \phi)$ defines a chain contraction of $\mathcal{C}$ to $\mathcal{H}$ over $\mathbf{Z} / p$.

Property (ii) follows that the homology groups of $\mathcal{C}$ and $\mathcal{H}$, with coefficients over $\mathbf{Z} / p$, are isomorphic.

Example 3.1. Let $K$ be the simplicial complex shown in Fig. 5. The set $\left((K, \partial), H_{K}, f_{K}, g_{K}, \phi_{K}\right)$ is an AT-model for $K$ over $\mathbf{Z} / 2$, where

$$
K=\left\{v_{1}, v_{2}, v_{3}, v_{4}, v_{1} v_{4}, v_{1} v_{2}, v_{2} v_{4}, v_{3} v_{4}, v_{2} v_{3}, v_{1} v_{2} v_{4}\right\}, H_{K}=\left\{v_{4}, v_{2} v_{3}\right\}
$$

and the images of the maps $f_{K}, g_{k}$ and $\phi_{K}$ are:

\begin{tabular}{c|c|c|c|c|c|c|c|c|c|c} 
& $v_{1}$ & $v_{2}$ & $v_{3}$ & $v_{4}$ & $v_{1} v_{4}$ & $v_{1} v_{2}$ & $v_{2} v_{4}$ & $v_{3} v_{4}$ & $v_{2} v_{3}$ & $v_{1} v_{2} v_{4}$ \\
\hline$f_{K}$ & $v_{4}$ & $v_{4}$ & $v_{4}$ & $v_{4}$ & 0 & 0 & 0 & 0 & $v_{2} v_{3}$ & 0 \\
$g_{K}$ & & & $v_{4}$ & & & & & $\alpha$ & & \\
$\phi_{K}$ & $v_{1} v_{4}$ & $v_{1} v_{2}+v_{1} v_{4}$ & $v_{3} v_{4}$ & 0 & 0 & $v_{1} v_{2} v_{4}$ & 0 & 0 & 0 & 0
\end{tabular}

where $\alpha=v_{2} v_{3}+v_{2} v_{4}+v_{3} v_{4}$. Then, the homology groups of $\mathcal{C}(K)$ over the field $\mathbf{Z} / 2$ are $H_{0}(\mathcal{C}(K) ; \mathbf{Z} / 2)=\mathbf{Z} / 2, H_{1}(\mathcal{C}(K) ; \mathbf{Z} / 2)=\mathbf{Z} / 2$ and $H_{2}(\mathcal{C}(K) ; \mathbf{Z} / 2)=0$. The representative cycles of homology generators in $\operatorname{dim} 0$ and 1 are $v_{4}$ and $\alpha$, respectively.

The main problem of this tool is that from the homology obtained from an AT-model, it is not possible, in general, to get all the torsion information of the integer homology of the object under study (see Example 2.3). 

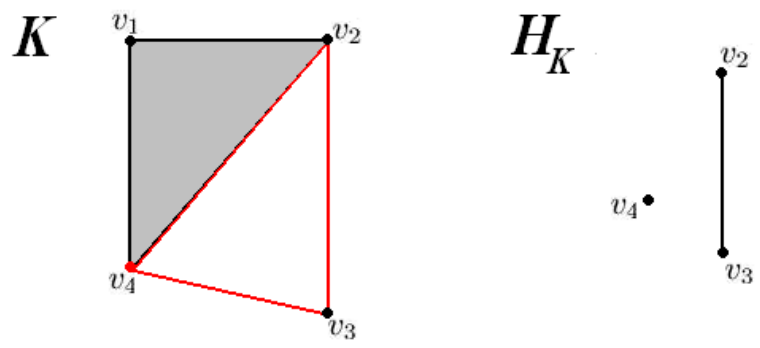

Figure 5: On the left, the simplicial complex $K$; in red, the representative cycles of homology generators of $H(\mathcal{C}(K) ; \mathbf{Z} / 2)$. On the right, the elements of $H_{K}$.

\section{The Notion of $\lambda$-AT-Model}

As far as we know all the algorithms in the literature for computing integer homology (with or without torsion) need to reduce the matrix of the differential to its SNF. Our aim is to compute integer homological information avoiding the computation of SNF. For this task, we first define the notion of $\lambda$ AT-model which can be seen as a generalization of the concept of AT-model, working in the integer domain. We study its properties and give an algorithm for computing it. Moreover, we will see that from a $\lambda$-AT-model for a chain complex $\mathcal{C}$, we can obtain the Betti numbers $\beta_{q}$, the prime numbers $p$ involved in the invariant factors of the torsion subgroup of $H(\mathcal{C} ; \mathbf{Z})$, the amount of invariant factors that are a power of $p$, and a set of representative cycles of generators of homology $\bmod p$, for each $p$. That is, from a $\lambda$-AT-model for a chain complex $\mathcal{C}$, we can obtain a set $\mathcal{I}$ of independent non-boundary cycles such that given any cycle $c$ of $\mathcal{C}, \lambda c$ can be expressed as a linear combination over $\mathbf{Z}$ of the cycles of $\mathcal{I}$. Finally, we present an algorithm for extracting this homological information, performed in $\mathcal{O}\left(m^{3} \psi(\lambda)\right)$ in the worst case, $\psi$ being the Euler function.

First of all, let us define the concept of $\lambda$-chain contraction as a generalization of chain contraction and study its properties. Later, we will define a $\lambda$-AT-model as a $\lambda$-chain contraction of a chain complex $\mathcal{C}$ to another chain complex with null differential.

Definition 4.1. Let $\lambda$ be an integer, $\lambda \neq 0$. A $\lambda$-chain contraction of a chain complex $\mathcal{C}$, to another chain complex $\mathcal{C}^{\prime}$ with differentials $d$ and $d^{\prime}$, respectively, is a set $(f, g, \phi, \lambda)$ such that: $f: \mathcal{C} \rightarrow \mathcal{C}^{\prime}$ and $g: \mathcal{C}^{\prime} \rightarrow \mathcal{C}$ are chain maps; $f g$ is $\lambda i d_{\mathcal{C}^{\prime}}$ and $\phi: \mathcal{C} \rightarrow \mathcal{C}$ is a chain homotopy of $\lambda i d_{\mathcal{C}^{\prime}}$ to $g f$, 
that is,

$$
f g=\lambda i d_{\mathcal{C}^{\prime}} \quad \text { and } \quad \lambda i d_{\mathcal{C}}-g f=\phi d+d \phi .
$$

Observe that a $\lambda$-chain contraction with $\lambda=1$ is a chain contraction. Moreover, if $(f, g, \phi, \lambda)$ is a $\lambda$-chain contraction of $\mathcal{C}$ to $\mathcal{C}^{\prime}$ with $\lambda=-1$ then $(-f, g,-\phi)$ is a chain contraction of $\mathcal{C}$ to $\mathcal{C}^{\prime}$.

Lemma 4.1. If $((C, d), H, f, g, \phi, \lambda)$ is a $\lambda$-AT-model, with $\lambda<0$, then $((C, d), H,-f, g,-\phi,-\lambda)$ is a $\lambda^{\prime}-A T$-model, with $\lambda^{\prime}:=-\lambda>0$.

If $(f, g, \phi, \lambda)$ is a $\lambda$-chain contraction of $\mathcal{C}$ to $\mathcal{C}^{\prime}$ and $\left(f^{\prime}, g^{\prime}, \phi^{\prime}, \lambda^{\prime}\right)$ is a $\lambda^{\prime}$-chain contraction of $\mathcal{C}^{\prime}$ to $\mathcal{C}^{\prime \prime}$, then

$$
\left(f^{\prime} f, g g^{\prime}, \lambda^{\prime} \phi+g \phi^{\prime} f, \lambda \lambda^{\prime}\right)
$$

is a $\lambda \lambda^{\prime}$-chain contraction of $\mathcal{C}$ to $\mathcal{C}^{\prime \prime}$. Another important property is the following.

Proposition 4.2. If there exists a $\lambda$-chain contraction $(f, g, \phi, \lambda)$ (with $\lambda \neq$ 0 ) of a chain complex $\mathcal{C}$ to another chain complex $\mathcal{C}^{\prime}$ then the free subgroups of $H(\mathcal{C} ; \mathbf{Z})$ and $H\left(\mathcal{C}^{\prime} ; \mathbf{Z}\right)$ are isomorphic. As a consequence, the Betti numbers of $\mathcal{C}$ and $\mathcal{C}^{\prime}$ coincide.

Proof. Let $d$ and $d^{\prime}$ be the differentials of $\mathcal{C}$ and $\mathcal{C}^{\prime}$, respectively. Let a be a representative cycle of a homology generator of the free subgroup of $H(\mathcal{C} ; \mathbf{Z})$, that is, $d(a)=0$ and for each $\mu \in \mathbf{Z}, \mu \neq 0, \mu a \notin \operatorname{Im} d$. Then $f(a)$ is a cycle of $\mathcal{C}^{\prime}$ since $f$ is a chain map and therefore $d^{\prime} f(a)=f d(a)=0$. Suppose that there exists an integer $\mu \neq 0$ and a chain $b^{\prime} \in \mathcal{C}^{\prime}$ such that $\mu f(a)=d^{\prime}\left(b^{\prime}\right)$. Then, $\lambda a-g f(a)=d \phi(a)$ since $(f, g, \phi, \lambda)$ is a $\lambda$-chain contraction and $a$ is a cycle. Therefore, $\lambda \mu a=d\left(g\left(b^{\prime}\right)+\mu \phi(a)\right)$ which is a contradiction since $a$ is a representative cycle of a homology generator of the free subgroup of $H(\mathcal{C} ; \mathbf{Z})$. Therefore $f(a)$ is a representative cycle of a homology generator of the free subgroup of $H\left(\mathcal{C}^{\prime} ; \mathbf{Z}\right)$.

Now, let $a$ and $b$ be representative cycles of two different homology generators of the free subgroup of $H(\mathcal{C} ; \mathbf{Z})$. Suppose that $f(a)$ and $f(b)$ are representative cycles of the same homology generator of the free subgroup of $H\left(\mathcal{C}^{\prime} ; \mathbf{Z}\right)$. Then there exists a chain $b^{\prime} \in \mathcal{C}^{\prime}$ such that $f(a)-f(b)=d^{\prime}\left(b^{\prime}\right)$. Then $\lambda(a-b)=d\left(g\left(b^{\prime}\right)+\phi(a-b)\right)$ which is a contradiction. Therefore, $f(a)$ and $f(b)$ are representative cycles of two different homology generators of the 
free subgroup of $H\left(\mathcal{C}^{\prime} ; \mathbf{Z}\right)$. Analogously, it is true that if $a^{\prime}$ and $b^{\prime}$ are representative cycles of two different homology generators of the free subgroup of $H\left(\mathcal{C}^{\prime} ; \mathbf{Z}\right)$ then $g\left(a^{\prime}\right)$ and $g\left(b^{\prime}\right)$ are representative cycles of two different homology generators of the free subgroup of $H(\mathcal{C} ; \mathbf{Z})$. We can conclude that the free subgroup of $H(\mathcal{C} ; \mathbf{Z})$ and $H\left(\mathcal{C}^{\prime} ; \mathbf{Z}\right)$ are isomorphic.

Now, define a $\lambda$-AT-model for a chain complex $\mathcal{C}$ as a $\lambda$-chain contraction of $\mathcal{C}$ to a chain complex with null differential.

Definition 4.2. Let $\mathcal{C}=(C, d)$ ) be a chain complex. Let $\lambda$ be a non-null integer. A $\lambda$-AT-model for $\mathcal{C}$ is a set $((C, d), H, f, g, \phi, \lambda)$, where

(i) $H=\left\{H_{q}\right\}$ generates a chain complex, denoted by $\mathcal{H}$, with null differential.

(ii) $(f, g, \phi, \lambda)$ defines a $\lambda$-chain contraction of $\mathcal{C}$ to $\mathcal{H}$.

As a consequence of Prop. 4.2 and since the differential of $\mathcal{H}$ is null, if there exists a $\lambda$-AT-model $((C, d), H, f, g, \phi, \lambda)$ for $\mathcal{C}$ then the free subgroup of $H(\mathcal{C} ; \mathbf{Z})$ is isomorphic to $\mathcal{H}$.

Proposition 4.3. Given a $\lambda$-AT-model, a rational AT-model (i.e., an ATmodel over Q) can directly be obtained as well as rational (co)homology and representative (co)cycles of (co)homology generators. Concretely, if $((C, d), H$, $f, g, \phi, \lambda)$ is a $\lambda$-AT-model for a chain complex $\mathcal{C}$, then $\left((C, d), H, \frac{1}{\lambda} f, g, \frac{1}{\lambda} \phi\right)$ is an AT-model for $\mathcal{C}$ over $\mathbf{Q}$ and $\{g(h): h \in H\}$ is a set of representative cycles of generators of $H(\mathcal{C} ; \mathbf{Q})$.

Universal Coefficient Theorem for Homology [14, pp. 332] states that each Betti number $\beta_{q}$ coincides with the vector space dimension of $H_{q}(\mathcal{C} ; \mathbf{Q})$. In addition, working in the integer domain, a $\lambda$-AT-model for $\mathcal{C}$ provides a set of $\beta_{q}$ independent non-boundary $q$-cycles of $\mathcal{C}$ over $\mathbf{Z}$, for each $q$.

Corollary 4.4. Let $((C, d), H, f, g, \phi, \lambda)$ be a $\lambda$-AT-model for a given chain complex $\mathcal{C}$. Then $\mathcal{H}$ is isomorphic to the free subgroup of $H(\mathcal{C} ; \mathbf{Z})$. Moreover, the set $\{g(h): h \in H\}$ is a set of independent non-boundary cycles of $\mathcal{C}$ over $\mathbf{Z}$ that generate the set $\{\lambda \alpha: \alpha \in F(\mathcal{C} ; \mathbf{Z})\}$.

Proof. Let $((C, d), H, f, g, \phi, \lambda)$ be a $\lambda$-AT-model for $\mathcal{C}$ and $\left\{h_{1}, \ldots, h_{m}\right\}$ a set of generators of $H$. Suppose that $\left\{g\left(h_{1}\right), \ldots, g\left(h_{m}\right)\right\}$ are not independent. 
Then there exists $i, 1 \leq i \leq m$, such that $g\left(h_{i}\right)=\sum_{j=1}^{m} c_{j} g\left(h_{j}\right)$ for some $c_{j} \in$ $\mathbf{Z}, 1 \leq j \neq i \leq m$, and $c_{i}=0$. Therefore $f g\left(h_{i}\right)=\sum_{j=1}^{m} c_{j} f g\left(h_{j}\right)$, so that $\lambda h_{i}=\sum_{j=1}^{m} c_{j} \lambda h_{j}$. Simplifying, $h_{i}=\sum_{j=1}^{m} c_{j} h_{j}$, which is a contradiction. Let $\alpha$ be a homology class of the free part of $H(\mathcal{C} ; \mathbf{Z})$ and $c$ a representative cycle of $\alpha$, that is, $\alpha=[c]$. Since $f(c) \in H$, then $f(c)=\sum_{i=1}^{m} c_{i} h_{i}, c_{i} \in \mathbf{Z}$. Therefore, $g f(c)=\sum_{i=1}^{m} c_{i} g\left(h_{i}\right)$. So, $\sum_{i=1}^{m} c_{i} g\left(h_{i}\right)=\lambda c-\phi d(c)-d \phi(c)$. Since $c$ is a cycle, then $\sum_{i=1}^{m} c_{i} g\left(h_{i}\right)=\lambda c-d \phi(c)$. Considering homology classes, $\sum_{i=1}^{m} c_{i}\left[g\left(h_{i}\right)\right]=\lambda[c]$. Consequently, $\left\{g\left(h_{1}\right), \ldots, g\left(h_{n}\right)\right\}$ is a set of independent non-boundary representative cycles of generators that generate the set $\{\lambda \alpha: \alpha \in F(\mathcal{C} ; \mathbf{Z})\}$.

Similar to rational AT-models, an AT-model over $\mathbf{Z} / p$ (where $p$ is a prime which does not divide $\lambda$ ) can always be defined from a $\lambda$-AT-model.

Proposition 4.5. Given a $\lambda$-AT-model $((C, d), H, f, g, \phi, \lambda)$ and a prime $p$ such that $p$ does not divide $\lambda$, then $\left(\left(C, d_{\mathbf{z} / p}\right), H, f_{\mathbf{z} / p}, g_{\mathbf{z} / p}, \phi_{\mathbf{z} / p}\right)$, where

$d_{\mathbf{z} / p}=d \bmod p, \quad f_{\mathbf{z} / p}=\lambda^{-1} f \bmod p, \quad g_{\mathbf{z} / p}=g \bmod p, \quad \phi_{\mathbf{z} / p}=\lambda^{-1} \phi \bmod p$,

is an AT-model for $\mathcal{C}$ over $\mathbf{Z} / p$. Moreover, $\left\{g_{\mathbf{z} / p}(h): h \in H\right\}$ is a set of representative cycles of generators of $H(\mathcal{C} ; \mathbf{Z} / p)$.

An important property of $\lambda$-AT-models is that we can obtain the prime numbers $p$ involved in the torsion subgroup of the homology of $\mathcal{C}$.

Proposition 4.6. Let $((C, d), H, f, g, \phi, \lambda)$ be a $\lambda$-AT-model. Let $a \in \mathcal{C}$ such that $d(a)=0$. If there exists $b \in \mathcal{C}$ such that $d(b)=\mu a$ where $\mu \in \mathbf{Z}, \mu \neq 0$ and for each $\rho$, where $0<\rho<\mu$, $\rho a \notin \operatorname{Im} d$, then $f(a)=0$ and $\mu$ divides $\lambda$.

Proof. Suppose that $b \in \mathcal{C}$ such that $d(b)=\mu a$ where $\mu \in \mathbf{Z}, \mu \neq 0$ and for each $\rho$, where $0<\rho<\mu, \rho a \notin \operatorname{Im} d$. First, let us prove that $f(a)=0$. Suppose that $f(a) \neq 0$. Then $\mu f(a) \neq 0$ (since the ground ring is $\mathbf{Z}$ ). On the other hand, $\mu f(a)=f(\mu a)=f(d(b))=0$, since $f$ is a chain map and the differential of $\mathcal{H}$ is null. We then have that $f(a)=0$ which is a contradiction. Now, suppose that $\mu$ does not divide $\lambda$, then there exist $s, r \in \mathbf{Z}$, such that $0<r<\mu$ and $\lambda=s \mu+r$. On one hand, $r a \notin \operatorname{Im} d$. On the other hand, $r a=(\lambda-s \mu) a=\lambda a-s \mu a=g f(a)+\phi d(a)+d \phi(a)-s d(b)=d(\phi(a)-s b) \in$ $\operatorname{Im} d$ which is a contradiction. We conclude that $\mu$ divides $\lambda$.

Corollary 4.7. Let $((C, d), H, f, g, \phi, \lambda)$ be a $\lambda$-AT-model. If $p^{t_{(q, p)}}$ is an invariant factor of $H_{q}(\mathcal{C} ; \mathbf{Z})$, then $p$ divides $\lambda$. 
Observe that if $\lambda$ is 'large' then there are lots of candidates to take part in the torsion subgroup of $H(\mathcal{C} ; \mathbf{Z}$ ) (all the primes that divide $\lambda$ ). Therefore, an important task could be to get the minimum $\lambda>0$ such that there exists a $\lambda$-AT-model for $\mathcal{C}$.

Proposition 4.8. Let $\mathcal{C}=(C, d)$ be a chain complex such that in each dim $q$ the matrix of $d_{q}$ coincides with its SNF. Let $\rho$ be the lowest common multiple of all the non null elements that appear in the matrices of $d_{q}$ for all $q$. Define the homomorphisms $f, g$ and $\phi$ and the set $H$ as follows:

- If $x \in C$ such that $d(x)=\mu y$ for some integer $\mu>0$ then

$$
f(x)=0, f(y)=0, \phi(x)=0 \text { and } \phi(y)=\frac{\rho}{\mu} x .
$$

- If $x \in C$ such that $d(x)=0$ and there is not any $z \in C$ being $d(z)=\mu x$ for some integer $\mu>0$, then

$$
f(x)=x, g(x)=\rho x \text { and } \phi(x)=0 .
$$

- $H=\{x \in C$ such that $d(x)=0$ and there is not any $z \in C$ such that $d(z)=\mu x$ for some integer $\mu>0\}$.

The set $((C, d), H, f, g, \phi, \rho)$ defines a $\rho$-AT-model. Moreover, this $\rho$-ATmodel satisfies that a prime $p$ divides $\rho$ if and only if it takes part in the torsion subgroup of $H(\mathcal{C} ; \mathbf{Z})$.

Observe that in order to obtain a $\lambda$-AT-model for a chain complex $\mathcal{C}$ with minimum $\lambda$, we need to compute the SNF of the matrix of the differential of $\mathcal{C}$ in each dimension. The following algorithm computes a $\lambda$-AT-model for a chain complex $\mathcal{C}$ without computing the SNF of the differential. In this case, $\lambda$ might be non-minimum.

Algorithm 4.9. Computing a $\lambda$-AT-model for a chain complex $\mathcal{C}$.

INPUT: A chain complex $\mathcal{C}=(C, d)$ of dim $n$.

$H_{0}:=C_{0}, \quad A:=C_{0}, \quad \lambda:=1, f_{0}:=i d_{C_{0}}, g_{0}:=i d_{H_{0}}, \phi_{0}:=0$.

For $q=1$ to $q=n$ do

$H_{q}:=\{\}, x:=0, \gamma:=0, \gamma^{\prime}:=0$. 
While $M:=\left\{\left|\left\langle f_{q-1} d_{q}(c), c^{\prime}\right\rangle\right| \neq 0: c \in C_{q}, c^{\prime} \in H_{q-1}\right\}$ is not empty do Take $\alpha \in H_{q-1}, \beta \in C_{q}$ s.t. $\left|\left\langle f_{q-1} d_{q}(\beta), \alpha\right\rangle\right|=\min M$ then

$$
\begin{aligned}
& H_{q-1}:=H_{q-1} \backslash\{\alpha\}, A:=A \cup\{\beta\}, f_{q}(\beta):=0, \phi_{q}(\beta):=0, \\
& x:=\left\langle f_{q-1} d_{q}(\beta), \alpha\right\rangle, \gamma:=f_{q-1} d_{q}(\beta), \gamma^{\prime}:=\lambda \beta-\phi_{q-1} d_{q}(\beta) . \\
& \text { For each } b \in C_{r}, 0 \leq r \leq q-1, \text { do } \\
& \quad f_{r}(b):=x f_{r}(b)-\left\langle f_{r}(b), \alpha\right\rangle \gamma, \\
& \quad \phi_{r}(b):=x \phi_{r}(b)+\left\langle f_{r}(b), \alpha\right\rangle \gamma^{\prime}, \\
& \lambda:=x \lambda .
\end{aligned}
$$

For each $a \in C_{q} \backslash A$ do

$$
\begin{aligned}
& H_{q}:=H_{q} \cup\{a\}, A:=A \cup\{a\}, \\
& f_{q}(a):=a, g_{q}(a):=\lambda a-\phi_{q-1} d_{q}(a), \phi_{q}(a):=0 .
\end{aligned}
$$

Output: The set $((C, d), H, f, g, \phi, \lambda)$.

Fixed $q$, there are two different parts in the algorithm. First, while the matrix $M$ corresponding to $f_{q-1} d_{q}$ is non-null, take $\alpha \in H_{q-1}$ and $\beta \in C_{q}$ such that $\left|\left\langle f_{q-1} d_{q}(\beta), \alpha\right\rangle\right|$ is the minimum of $M$ (without considering signs). Then, $\alpha$ is deleted from $H_{q-1}$ (i.e., a homology class is destroyed) and $\beta$ is added to $A$ (i.e, $\beta$ has been used). Second, if the new redefined matrix $M$ is null, add, to $H_{q}$, the non-used elements of $C_{q}$ (i.e. new classes of homology are created). At the end of the algorithm, the set $A$ consists in an ordered set of all the elements of $C$.

Theorem 4.10. The set $((C, d), H, f, g, \phi, \lambda)$ obtained applying Alg. 4.9 defines a $\lambda$-AT-model for the chain complex $\mathcal{C}=(C, d)$.

Proof. Let $A=\left\{a^{1}, \ldots, a^{m}\right\}$ such that if $i<j$ then $a^{i}$ was added to $A$ before $a^{j}$ when the algorithm was applied. Fixed $i$, assume that $f_{r-1} d_{r}\left(a^{k}\right)=0$, $\lambda a^{k}-g_{r} f_{r}\left(a^{k}\right)=\phi_{r-1} d_{r}\left(a^{k}\right)+d_{r+1} \phi_{r}\left(a^{k}\right), f_{r+1} \phi_{r}\left(a^{k}\right)=0$ and, if $a^{k} \in H_{r}$, $d_{r} g_{r}\left(a^{k}\right)=0$ and $f_{r} g_{r}\left(a^{k}\right)=\lambda a^{k}$, for $1 \leq k<i$ and $r=\operatorname{dim}\left(a^{k}\right)$.

Let $q=\operatorname{dim}\left(a^{i}\right)$. We have to prove that the redefined maps that we will denote here by $f^{\prime}, g^{\prime}$ and $\phi^{\prime}$ and the new integer $\lambda^{\prime}$ satisfy that $f_{r-1}^{\prime} d_{r}\left(a^{k}\right)=0$, $\lambda^{\prime} a^{k}-g_{r}^{\prime} f_{r}^{\prime}\left(a^{k}\right)=\phi_{r-1}^{\prime} d_{r}\left(a^{k}\right)+d_{r+1} \phi_{r}^{\prime}\left(a^{k}\right), f_{r+1}^{\prime} \phi_{r}^{\prime}\left(a^{k}\right)=0$ and, if $a^{k} \in H_{r}$, $d_{r} g_{r}^{\prime}\left(a^{k}\right)=0$ and $f_{r}^{\prime} g_{r}^{\prime}\left(a^{k}\right)=\lambda^{\prime} a^{k}$ for $1 \leq k \leq i$ and $r=\operatorname{dim}\left(a^{k}\right)$.

We have to consider two cases. (1) If $a^{i}$ was added to $A$ while $M$ was non-empty, then $g^{\prime}=g$ (that is, $g$ does not change). Therefore, if $a^{k} \in H_{r}$ for some $r$, then $d_{r} g_{r}^{\prime}\left(a^{k}\right)=d_{r} g_{r}\left(a^{k}\right)=0$ for all $k, 1 \leq k<i$. Consider the element $\alpha \in H_{q-1}$ that defines the auxiliary parameter $x=\left\langle f_{q-1} d_{q}\left(a^{i}\right), \alpha\right\rangle$ at this stage. 
If $k=i$, then $a^{i} \notin H_{q}$. We have that $f_{q-1}^{\prime} d_{q}\left(a^{i}\right)=x f_{q-1} d_{q}\left(a^{i}\right)-$ $\left\langle f_{q-1} d_{q}\left(a^{i}\right), \alpha\right\rangle \gamma=x \gamma-x \gamma=0$ and $\phi_{q-1}^{\prime} d_{q}\left(a^{i}\right)+d_{q+1} \phi_{q}^{\prime}\left(a^{i}\right)=\phi_{q-1}^{\prime} d_{q}\left(a^{i}\right)=$ $x \phi_{q-1} d_{q}\left(a^{i}\right)+\left\langle f_{q-1} d_{q}\left(a^{i}\right), \alpha\right\rangle \gamma^{\prime}=x\left(\phi_{q-1} d_{q}\left(a^{i}\right)+\lambda a^{i}-\phi_{q-1} d_{q}\left(a^{i}\right)\right)=x \lambda a^{i}=$ $\lambda^{\prime} a^{i}$. Moreover, $f_{q+1}^{\prime} \phi_{q}^{\prime}\left(a^{i}\right)=0$ since $\phi_{q}^{\prime}\left(a^{i}\right)=0$.

Observe that: For any $r$-chain $b$ such that $r<q-1, f_{r}^{\prime}(b)=x f_{r}(b)$ and $\phi_{r}^{\prime}(b)=x \phi_{r}(b) . \quad d_{q}\left(\gamma^{\prime}\right)=d_{q}\left(\lambda a^{i}-\phi_{q-1} d_{q}\left(a^{i}\right)\right)=\lambda d_{q}\left(a^{i}\right)-\left(\lambda d_{q}\left(a^{i}\right)-\right.$ $\left.g_{q-1} f_{q-1} d_{q}\left(a^{i}\right)-\phi_{q-1} d_{q-1} d_{q}\left(a^{i}\right)\right)=g_{q-1} f_{q-1} d_{q}\left(a^{i}\right)=g_{q-1}(\gamma)$. For any $q$-chain $b, f_{q}(b)=0$ and $\phi_{q}(b)=0$, therefore, $f_{q}^{\prime}(b)=0$ and $\phi_{q}^{\prime}(b)=0$, since $a^{i}$ was added to $A$ while $M$ was non-empty.

For $1 \leq k<i$. (1.a) If $r:=\operatorname{dim}\left(a^{k}\right)<q-1$ then $f_{r-1}^{\prime} d_{r}\left(a^{k}\right)=$ $x f_{r-1} d_{r}\left(a^{k}\right)=0$ by induction and $\phi_{r-1}^{\prime} d_{r}\left(a^{k}\right)+d_{r+1} \phi_{r}^{\prime}\left(a^{k}\right)=x\left(\phi_{r-1} d_{r}\left(a^{k}\right)+\right.$ $\left.d_{r+1} \phi_{r}\left(a^{k}\right)\right)=x\left(\lambda a^{k}-g_{r} f_{r}\left(a^{k}\right)\right)=\lambda^{\prime} a^{k}-g_{r} f_{r}^{\prime}\left(a^{k}\right)$. Moreover, $f_{r+1}^{\prime} \phi_{r}^{\prime}\left(a^{k}\right)=$ $x f_{r+1}^{\prime} \phi_{r}\left(a^{k}\right)=x\left(x f_{r+1} \phi_{r}\left(a^{k}\right)-\left\langle f_{r+1} \phi_{r}\left(a^{k}\right), \alpha\right\rangle \gamma\right)=0$ by induction. If $a^{k} \in H_{r}$ then $f_{r}^{\prime} g_{r}\left(a^{k}\right)=x f_{r} g_{r}\left(a^{k}\right)=x \lambda a^{k}=\lambda^{\prime} a^{k}$. (1.b) If $\operatorname{dim}\left(a^{k}\right)=q-1$, then $f_{q-2}^{\prime} d_{q-1}\left(a^{k}\right)=x f_{q-2} d_{q-1}\left(a^{k}\right)=0$ by induction and $\phi_{q-2}^{\prime} d_{q-1}\left(a^{k}\right)+$ $d_{q} \phi_{q-1}^{\prime}\left(a^{k}\right)=x \phi_{q-2} d_{q-1}\left(a^{k}\right)+d_{q}\left(x \phi_{q-1}\left(a^{k}\right)+\left\langle f_{q-1}\left(a^{k}\right), \alpha\right\rangle \gamma^{\prime}\right)=x\left(\phi_{q-2} d_{q-1}\left(a^{k}\right)+\right.$ $\left.d_{q} \phi_{q-1}\left(a^{k}\right)\right)+\left\langle f_{q-1}\left(a^{k}\right), \alpha\right\rangle d_{q}\left(\gamma^{\prime}\right)=x\left(\lambda a^{k}-g_{q-1} f_{q-1}\left(a^{k}\right)\right)+\left\langle f_{q-1}\left(a^{k}\right), \alpha\right\rangle g_{q-1}(\gamma)=$ $\lambda^{\prime} a^{k}-g_{q-1} f_{q-1}^{\prime}\left(a^{k}\right)$. Moreover, $f_{q}^{\prime} \phi_{q-1}^{\prime}\left(a^{k}\right)=f_{q}^{\prime}\left(x \phi_{q-1}\left(a^{k}\right)+\left\langle f_{q-1}\left(a^{k}\right), \alpha\right\rangle \gamma^{\prime}\right)$. Now, $f_{q}^{\prime} \phi_{q-1}\left(a^{k}\right)=0$ since for any $q$-chain $b, f_{q}^{\prime}(b)=0 ; f_{q}^{\prime}\left(\gamma^{\prime}\right)=f_{q}^{\prime}\left(\lambda a^{i}-\right.$ $\left.\phi_{q-1} d_{q}\left(a^{i}\right)\right)=0$ for the same reason than above. Then, $f_{q}^{\prime} \phi_{q-1}^{\prime}\left(a^{k}\right)=0$. If $a^{k} \in H_{q-1}$ then $a^{k} \neq \alpha$ and $f_{q-1}^{\prime} g_{q-1}\left(a^{k}\right)=x f_{q-1} g_{q-1}\left(a^{k}\right)-\left\langle f_{q-1} g_{q-1}\left(a^{k}\right), \alpha\right\rangle \gamma=$ $x \lambda a^{k}-\left\langle\lambda a^{k}, \alpha\right\rangle \gamma=x \lambda a^{k}=\lambda^{\prime} a^{k}$. (1.c) If $\operatorname{dim}\left(a^{k}\right)=q$, then $f_{q-1}^{\prime} d_{q}\left(a^{k}\right)=$ $x f_{q-1} d_{q}\left(a^{k}\right)-\left\langle f_{q-1} d_{q}\left(a^{k}\right), \alpha\right\rangle \gamma=0$ by induction and $\phi_{q-1}^{\prime} d_{q}\left(a^{k}\right)+d_{q+1} \phi_{q}^{\prime}\left(a^{k}\right)=$ $x\left(\phi_{q-1} d_{q}\left(a^{k}\right)+d_{q+1} \phi_{q}\left(a^{k}\right)\right)+\left\langle f_{q-1} d_{q}\left(a^{k}\right), \alpha\right\rangle \gamma^{\prime}=x\left(\lambda a^{k}-g_{q} f_{q}\left(a^{k}\right)\right)=\lambda^{\prime} a^{k}-$ $g_{q} f_{q}^{\prime}\left(a^{k}\right)$. Moreover, $f_{q+1}^{\prime} \phi_{q}^{\prime}\left(a^{k}\right)=0$.

(2) If $a^{i}$ was added to $A$ when $M$ was empty, then $\lambda^{\prime}=\lambda$ (that is, $\lambda$ does not change). First, $f_{q-1}^{\prime} d_{q}\left(a^{i}\right)=f_{q-1} d_{q}\left(a^{i}\right)=0$ by induction and $\lambda a^{i}-$ $g_{q}^{\prime} f_{q}^{\prime}\left(a^{i}\right)=\lambda a^{i}-g_{q}^{\prime}\left(a^{i}\right)=\lambda a^{i}-\left(\lambda a^{i}-\phi_{q-1} d_{q}\left(a^{i}\right)\right)=\phi_{q-1} d_{q}\left(a^{i}\right)=\phi_{q-1}^{\prime} d_{q}\left(a^{i}\right)=$ $\phi_{q-1}^{\prime} d_{q}\left(a^{i}\right)+d_{q+1} \phi_{q}^{\prime}\left(a^{i}\right)$. Second, $d_{q} g_{q}^{\prime}\left(a^{i}\right)=\lambda d_{q}\left(a^{i}\right)-d_{q} \phi_{q-1} d_{q}\left(a^{i}\right)=\lambda d_{q}\left(a^{i}\right)-$ $\left(\lambda d_{q}\left(a^{i}\right)-g_{q-1} f_{q-1} d_{q}\left(a^{i}\right)-\phi_{q_{2}} d_{q-1} d_{q}\left(a^{i}\right)\right)=g_{q-1} f_{q-1} d_{q}\left(a^{i}\right)=0$ and $f_{q}^{\prime} g_{q}^{\prime}\left(a^{i}\right)=$ $f_{q}^{\prime}\left(\lambda a^{i}-\phi_{q-1} d_{q}\left(a^{i}\right)\right)=\lambda a^{i}-f_{q} \phi_{q-1} d_{q}\left(a^{i}\right)=\lambda a^{i}$, since $f_{r+1} \phi_{r}\left(a^{k}\right)=0$ for $1 \leq k<i$ and $r=\operatorname{dim}\left(a^{k}\right)$, by induction. Finally, $f_{q+1}^{\prime} \phi_{q}^{\prime}\left(a^{i}\right)=0$ since $\phi_{q}^{\prime}\left(a^{i}\right)=0$.

To study the complexity, consider the order of the elements of $C$ given by the set $A$. Fixed $i$, count the number of elementary operations involved. We have to update $f_{r}(b)$ and $\phi_{r}(b)$ for each $b \in C_{r}, 0 \leq r \leq q-1$. Observe that in order to update $f_{r}(b)$ and $\phi_{r}(b)$ in the worst case, we have to add to both a chain containing $m_{q}$ generators (if $m_{q}$ is the number of generators of 


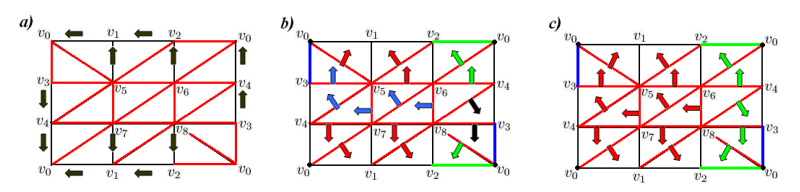

Figure 6: A visual interpretation of the maps $f_{S}$ and $\phi_{S}$ on a triangulation of the Klein bottle when applying Alg. 4.9. a) after adding all the vertices and edges; b) after adding all the vertices edges and triangles except for $v_{4} v_{6} v_{8} ; \mathrm{c}$ ) at the end.

$\operatorname{dim} q$ ). In the worst case, $m_{q}$ could be of the same order than $m$ (the total number of generators of $\mathcal{C}$ ). So, fixed $i$, the total cost of these operations is $O\left(m^{2}\right)$. Therefore, the total algorithm runs in time at most $O\left(m^{3}\right)$.

Example 4.11. Consider the simplicial complex $S$ derived from the triangulation of the Klein bottle given in Fig. 因 and the chain complex $\mathcal{C}(S)$ of dim 2 associated to S. Applying Alg. 4.9, we obtain a 2-AT-model, $\left(C(S), H_{S}, f_{S}, g_{S}, \phi_{S}, 2\right)$, for $\mathcal{C}(S)$. For the sake of simplicity in this example, each time a negative value for $\lambda$ appears, we apply Lemma 4.1 and turn it into positive. After running Alg. 4.9 only for the vertices and edges of $S$, we get that, at this stage, $H_{S}=\left\{v_{0}\right\} \cup\{x: x$ is an edge marked in red in Fig. 因a)\} and the values of $f_{S}, g_{S}$ and $\phi_{S}$ at this stage are:

\begin{tabular}{c|c|c|c|c} 
& $v_{0}$ & $v_{i}$ & $y$ & $x$ \\
\hline$f_{S}$ & $v_{0}$ & $v_{0}$ & 0 & $x$ \\
$g_{S}$ & $v_{0}$ & & & $x-\phi_{S} \partial_{1}(x)$ \\
$\phi_{S}$ & 0 & $\gamma_{\left(v_{0}, v_{i}\right)}$ & 0 & 0
\end{tabular}

where $1 \leq i \leq 8, y$ denotes an edge drawn in black in Fig. 因a) and $\gamma_{\left(v_{0}, v_{i}\right)}$ is a path connecting the vertices $v_{0}$ and $v_{i}, i \neq 0$ following the arrows drawn in black in Fig. 因a); for example, $\gamma_{\left(v_{0}, v_{6}\right)}=v_{0} v_{1}+v_{1} v_{2}+v_{2} v_{6}$.

Now, apply the algorithm for all the vertices, edges and triangles except for the triangle $v_{4} v_{6} v_{8}$ (to get a better idea of the geometric meaning of the maps $f_{S}, g_{S}$ and $\phi_{S}$, suppose that we do not consider this triangle until the end). We get that $H_{S}=\left\{v_{0}, v_{0} v_{2}, v_{0} v_{3}\right\}$. The values of $f_{S}, g_{S}$ and $\phi_{S}$ at this stage are:

\begin{tabular}{c|c|c|c|c|c|c|c} 
& $v_{0}$ & $v_{i}$ & $y$ & $v_{0} v_{2}$ & $v_{0} v_{3}$ & $z$ & $t$ \\
\hline$f_{S}$ & $v_{0}$ & $v_{0}$ & 0 & $v_{0} v_{2}$ & $v_{0} v_{3}$ & $\gamma_{(z)}^{\prime}$ & 0 \\
$g_{S}$ & $v_{0}$ & & & $v_{0} v_{2}-v_{1} v_{2}-v_{0} v_{1}$ & $v_{0} v_{3}+v_{3} v_{4}-v_{0} v_{4}$ & & \\
$\phi_{S}$ & 0 & $\gamma_{\left(v_{0}, v_{i}\right)}$ & 0 & 0 & 0 & $\gamma_{(z)}^{\prime \prime}$ & 0
\end{tabular}


where $z$ denotes one red edge (see Fig. 4.b)) and $t$ a triangle except for $v_{4} v_{6} v_{8}$. $\gamma_{z}^{\prime}$ is $0, \pm v_{0} v_{3}, \pm v_{0} v_{2}$ or $v_{0} v_{2}-v_{0} v_{3}$ if the arrow drawn over $z$ in Fig. 4. b) is, respectively, red, blue, green or black. $\gamma_{(z)}^{\prime \prime}$ is a 'path' from $z$ following the arrows drawn in Fig. 4.b). For example, $\gamma_{\left(v_{6} v_{7}\right)}=v_{5} v_{6} v_{7}-v_{2} v_{5} v_{6}+v_{1} v_{2} v_{5}+$ $v_{4} v_{5} v_{7}-v_{3} v_{4} v_{5}-v_{0} v_{3} v_{5}+v_{0} v_{1} v_{5}+v_{0} v_{4} v_{7}-v_{0} v_{1} v_{7}$.

Now, add the triangle $v_{4} v_{6} v_{8}$. Then $f_{S} \partial\left(v_{4} v_{6} v_{8}\right)$ is $2 v_{0} v_{3}$. Therefore, the new images for $f_{S}, g_{S}$ and $\phi_{S}$ are:

\begin{tabular}{c|c|c|c|c|c|c|c|c} 
& $v_{0}$ & $v_{i}$ & $y$ & $v_{0} v_{2}$ & $v_{0} v_{3}$ & $z$ & $t$ & $v_{4} v_{6} v_{8}$ \\
\hline$f_{S}$ & $2 v_{0}$ & $2 v_{0}$ & 0 & $2 v_{0} v_{2}$ & 0 & $2 \gamma_{(z)}^{\prime \prime \prime}$ & 0 & 0 \\
$g_{S}$ & $v_{0}$ & & & $v_{0} v_{2}-v_{1} v_{2}-v_{0} v_{1}$ & & & \\
$\phi_{S}$ & 0 & $2 \gamma_{\left(v_{0}, v_{i}\right)}$ & 0 & 0 & $2 v_{4} v_{6} v_{8}$ & $2 \gamma_{(z)}^{\prime v}$ & 0 & 0
\end{tabular}

where $\gamma_{z}^{\prime \prime \prime}$ is 0 or $\pm v_{0} v_{2}$ if the arrow drawn over $z$ in Fig. 4. c) is, respectively, red or green. $\gamma_{(z)}^{\prime v}$ is a 'path' from $z$ following the arrows drawn in Fig. 4.c).

The following proposition shows that AT-models over $\mathbf{Z} / p$, where $p$ is prime, can also be computed using Alg. 4.9.

Proposition 4.12. Working with coefficients in $\mathbf{Z} / p$, where $p$ is prime, let

$$
\left.\left(C, d_{\mathbf{z} / p}\right), H_{\mathbf{z} / p}, f_{\mathbf{z} / p}, g_{\mathbf{z} / p}, \phi_{\mathbf{z} / p}, \lambda\right)
$$

be the output of Alg. 4.9. Then

$$
\left(\left(C, d_{\mathbf{z} / p}\right), H_{\mathbf{z} / p}, \lambda^{-1} f_{\mathbf{z} / p}, g, \lambda^{-1} \phi_{\mathbf{z} / p}\right)
$$

is an AT-model over $\mathbf{Z} / p$. Furthermore, $\left\{g_{\mathbf{z} / p}(h): h \in H_{\mathbf{z} / p}\right\}$ is a set of representative cycles of generators of $H(\mathcal{C} ; \mathbf{Z} / p)$.

In order to obtain a $\lambda$-AT-model with $\lambda$ 'small', the following algorithm can be used as a preprocessing of Alg. 4.9. It only considers the case in which $\left\langle d\left(\alpha^{\prime}\right), \alpha\right\rangle=1$. The output of this algorithm is a chain contraction of the given chain complex $\mathcal{C}$ to another chain complex $\mathcal{C}^{\prime}$ with less number of generators. We can compute a $\lambda$-AT-model for $\mathcal{C}^{\prime}$ applying Alg. 4.9 to $\mathcal{C}^{\prime}$. We can obtain a $\lambda$-AT model for $\mathcal{C}$, composing the chain contraction of $\mathcal{C}$ to $\mathcal{C}^{\prime}$ with the $\lambda$-AT-model for $\mathcal{C}^{\prime}$ obtained before, using Formula (1) given in page 11 .

Algorithm 4.13. Preprocessing. 
INPUT: A chain complex $\mathcal{C}=(C, d)$ of $\operatorname{dim} n$.

$C_{0}^{\prime}:=C_{0}, \quad d_{0}^{\prime}=d_{0}, \quad A:=C_{0}, \quad f_{0}:=i d_{C_{0}}, g_{0}:=i d_{C_{0}^{\prime}}, \quad \phi_{0}:=0$.

For $q=1$ to $q=n$ do

$C_{q}^{\prime}:=\{\}, x:=0, \gamma:=0, \gamma^{\prime}:=0$.

While $\min \left\{\left|\left\langle f_{q-1} d_{q}(c), c^{\prime}\right\rangle\right| \neq 0: c \in C_{q}, c^{\prime} \in C_{q-1}^{\prime}\right\}=1$ do

Take $\alpha \in C_{q-1}^{\prime}, \beta \in C_{q}$ s.t. $\left|\left\langle f_{q-1} d_{q}(\beta), \alpha\right\rangle\right|=1$ then

$C_{q-1}^{\prime}:=C_{q-1}^{\prime} \backslash\{\alpha\}, A:=A \cup\{\beta\}, f_{q}(\beta):=0, \phi_{q}(\beta):=0$,

$x:=\left\langle f_{q-1} d_{q}(\beta), \alpha\right\rangle, \gamma:=f_{q-1} d_{q}(\beta), \gamma^{\prime}:=\beta-\phi_{q-1} d_{q}(\beta)$.

For each $b \in C_{q-1}$ do

$$
\begin{aligned}
& f_{q-1}(b):=f_{q-1}(b)-x\left\langle f_{q-1}(b), \alpha\right\rangle \gamma, \\
& \phi_{q-1}(b):=\phi_{q-1}(b)+x\left\langle f_{q-1}(b), \alpha\right\rangle \gamma^{\prime},
\end{aligned}
$$

For each $a \in C_{q} \backslash A$ do

$$
\begin{aligned}
& C_{q}^{\prime}:=C_{q}^{\prime} \cup\{a\}, A:=A \cup\{a\}, \\
& d_{q}^{\prime}(a):=f_{q-1} d_{q}(a), \quad f_{q}(a):=a, g_{q}(a):=a-\phi_{q-1} d_{q}(a), \phi_{q}(a):=0 .
\end{aligned}
$$

Output: The set $\left((C, d),\left(C^{\prime} . d^{\prime}\right), f, g, \phi, \lambda\right)$.

Theorem 4.14. The set $\left((C, d),\left(C^{\prime}, d^{\prime}\right), f, g, \phi\right)$ obtained applying Alg. 4.13 defines a chain contraction of the chain complex $\mathcal{C}=(C, d)$ to the chain complex $\mathcal{C}^{\prime}=\left(C^{\prime}, d^{\prime}\right)$.

The proof of this theorem is given in the appendix.

\section{Extracting Integer Homological Information}

As showed before, a $\lambda$-AT-model for a given chain complex $\mathcal{C}$ provides information of the free subgroup of $H(\mathcal{C} ; \mathbf{Z})$ as well as the prime numbers $p$ involved in the invariant factors of $H(\mathcal{C} ; \mathbf{Z})$. In order to obtain representative cycles mod $p$ of generators of the free and the torsion subgroups of $H(\mathcal{C} ; \mathbf{Z})$ we have to compute AT-models for $\mathcal{C}$ over $\mathbf{Z} / p$, for each prime $p$ dividing $\lambda$.

Algorithm 5.1. Computing integer homology information and representative cycles of homology generators of a chain complex $\mathcal{C}$.

INPUT: a chain complex $\mathcal{C}=(C, d)$ of $\operatorname{dim} n$.

Apply Alg. 4.9 with coefficients in $\mathrm{Z}$ for computing a 


$$
\begin{aligned}
& \lambda \text {-AT-model }((C, d), H, f, g, \phi, \lambda) \text { for } \mathcal{C} ; \\
& \text { For } q=0 \text { to } q=n \text { do } \\
& \quad \beta_{q}:=\text { number of elements of } H_{q} ; \\
& G:=\{g(h): h \in H\} . \\
& \text { For each prime } p \text { dividing } \lambda \text { do }
\end{aligned}
$$

Apply Alg. 4.9 with coefficients in $\mathbf{Z} / p$ to compute an

$$
\begin{aligned}
& \text { AT-model for } \mathcal{C} \text { over } \mathbf{Z} / p: \quad\left(\left(C, d_{\mathbf{z} / p}\right), H_{\mathbf{z} / p}, f_{\mathbf{z} / p}, g_{\mathbf{z} / p}, \phi_{\mathbf{Z} / p}\right) \\
& \beta_{(q, p)}:=\text { number of elements of } H_{\mathbf{z} / p} \text { in } \operatorname{dim} q . \\
& T_{(0, p)}=\beta_{(0, p)}-\beta_{0} ; \\
& \text { For } q=1 \text { to } n \text { do } \\
& \quad T_{(q, p)}=\beta_{(q, p)}-\beta_{q}-T_{(q-1, p)}, \\
& \quad G_{\mathbf{Z} / p}=\left\{g_{\mathbf{z} / p}(h): h \in H_{\mathbf{z} / p}\right\} \\
& \text { The sets } G,\left\{\beta_{1}, \ldots, \beta_{n}\right\} \\
& \left\{G_{\mathbf{Z} / p}: p \text { is a prime dividing } \lambda\right\} \\
& \text { and }\left\{T_{(q, p)}: 0 \leq q \leq n \text { and } p \text { is a prime dividing } \lambda\right\} .
\end{aligned}
$$

Output: The sets $G,\left\{\beta_{1}, \ldots, \beta_{n}\right\}$,

After computing Alg. 5.1 for a given chain complex $\mathcal{C}$, we obtain:

- the Betti numbers $\beta_{q}$ for $0 \leq q \leq n$, and a set $G$ of independent nonboundary cycles of $\mathcal{C}$ over $\mathbf{Z}$ (in fact, $G$ is also a set of generators of $H(\mathcal{C} ; \mathbf{Q}))$;

- the prime numbers $p$ involved in the invariant factors corresponding to the torsion subgroup of $H(\mathcal{C}, \mathbf{Z})$, the amount of invariant factors in each $\operatorname{dim} q$ that are a power of $p, T_{(q, p)}$, and a set $G_{\mathbf{z} / p}$ of representative cycles of generators of $H(\mathcal{C} ; \mathbf{Z} / p)$, for each prime $p$ dividing $\lambda$.

This algorithm uses Alg. 4.9 over $\mathbf{Z}$ for computing a $\lambda$-AT-model for $\mathcal{C}$ and over $\mathbf{Z} / p$ for computing AT-models for $\mathcal{C}$, for each prime $p$ dividing $\lambda$. Since the complexity of Alg. 4.9 is $O\left(\mathrm{~m}^{3}\right)$, then the complexity of Alg. 5.1 is $O\left(m^{3} \psi(\lambda)\right)$ in the worst case, $\psi$ being the Euler function.

Example 5.2. In Example 4.11, we have applied Alg. 4.9 and computed a 2-AT-model for $\mathcal{C}(S)$. We obtained that $H_{S}=\left\{v_{0}, v_{0} v_{2}\right\}$ and then the Betti numbers of $\mathcal{C}$ are $\beta_{0}=1, \beta_{1}=1$ and $\beta_{2}=0$. Now, apply Alg. 4.9 with coefficients in $\mathbf{Z} / 2$ to obtain an AT-model $\left.\left(\left(C, d_{\mathbf{z} / 2}\right)\right), H_{\mathbf{z} / 2}, f_{\mathbf{z} / 2}, g_{\mathbf{z} / 2}, \phi_{\mathbf{z} / 2}\right)$ where $H_{\mathbf{z} / 2}=\left\{v_{0}, v_{0} v_{2}, v_{0} v_{4}, v_{0} v_{3} v_{8}\right\}$ for $\mathcal{C}(S)$. Then, $\beta_{(0,2)}=1, \beta_{(1,2)}=2$, $\beta_{(2,2)}=1$. Therefore, $T_{(0,2)}=0, T_{(1,2)}=1$ and $T_{(2,2)}=0$.

We conclude that $H_{0}(S ; \mathbf{Z}) \simeq \mathbf{Z}$ and $H_{1}(S ; \mathbf{Z}) \simeq \mathbf{Z} \oplus \mathbf{Z} / 2$. 

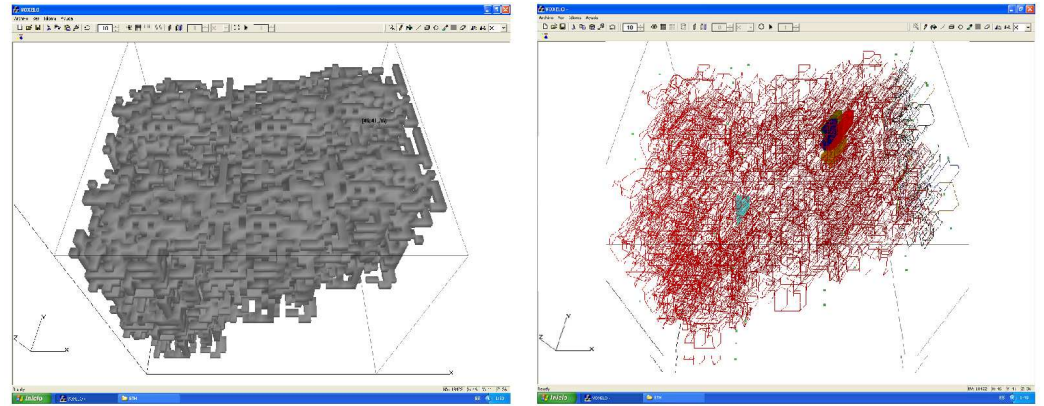

Figure 7: A porous 3D digital image and representative cycles of its 2709 black holes.

Alg. 5.1 has been implemented [9]. Fig. 5] shows a 3D digital image with 18491 black voxels. In 144 seconds we obtain that the image has 51 black connected components, 2709 black holes and 8 black cavities.

\section{Conclusions and Future Work}

We have constructed an algebraic tool, called $\lambda$-AT-model, in order to compute topological features of $n$-dimensional objects (for instance, $\mathrm{nD}$ digital pictures). Associating an algebraic representation to the initial object, a chain complex, the algorithm presented here provides the free subgroup of homology, with integer coefficients, as well as the primes $p$ that are the candidates to be involved in the torsion subgroup. In fact, such a set of candidates is the one of divisors of $\lambda$. We have determined the minimum $\lambda$ valid for the existence of a $\lambda$-AT-model. We have also presented an algorithm which gives a $\lambda$-AT-model with 'smaller' $\lambda$ than the one given in [10]. As for the complexity, the algorithm presented is performed in $O\left(m^{3} \psi(\lambda)\right)$ in the worst case, $\psi$ being the Euler function and $m$ being the number of cells of a cell decomposition of the object. In order to improve the complexity, first compute a chain contraction from the initial chain complex using Alg. 4.13 to a 'smaller' one with same integer homology and then compute the $\lambda$-ATmodel for the latter. Afterwards, in order to obtain the amount of invariant factors that are a power of $p$ and representative cycles mod $p$ of homology generators, for each $p$ we compute an AT-model with coefficients in $\mathbf{Z} / p$.

Future work can be addressed to obtain generators of the torsion subgroup of $H(\mathcal{C} ; \mathbf{Z})$, with integer coefficients. Another task is to study the computation of cohomology features over $\mathbf{Z}$ from a $\lambda$-AT-model. 


\section{References}

[1] Dahmen W., Micchelli, C.A.: On the Linear Independence of Multivariate $b$-splines I. Triangulation of simploids. SIAM J. Numer. Anal. v. 19, 993-1012 (1982)

[2] Dumas J.G., Saunders B., Villard G.: On Efficient Sparse Integer Matrix Smith Normal Form Computation. J. Sym. Comp. v. 32, 71-99 (2001)

[3] Gui X., Havas G.: On the Worst-case Complexity of Integer Gaussian Elimination. Proc. of the 1997 International Symposium on Symbolic and Algebraic Computation, Kihei, Maui, Hawaii, United States, 28-31 (1997)

[4] Gonzalez-Diaz R., Real P.: Computation of Cohomology Operations on Finite Simplicial Complexes. Homology, Homotopy and Applications, v. 5 (2), 83-93 (2003)

[5] Gonzalez-Diaz R., Real P.: Towards Digital Cohomology. Proc. of the 2003 International Conference on Discrete Geometry for Computer Imagery, Naples, Italy, November 19-21, LNCS v. 2886, 92-101 (2003)

[6] Gonzalez-Diaz R., Real P.: On the Cohomology of 3D Digital Images. Discrete Applied Math v. 147, 245-263 (2005)

[7] Gonzalez-Diaz R., Medrano B., Real P., Sanchez-Pelaez J.: Reusing Integer Homology Information of Binary Digital Images. Proc. of the 2006 International Conference on Discrete Geometry for Computer Imagery, Szeged, Hungary, October 25-27, LNCS v. 4245, 199-210 (2006)

[8] Gonzalez-Diaz R., Jimenez M.J., Medrano B., Real P.: Chain homotopies for Object Topological Representations. Discrete Applied Math (in press)

[9] Gonzalez-Diaz R., Sanchez-Pelaez J., Real P.: VOXELO Visualizing Topology Computations on Binary Voxel Volumes. Electronic Journal Imagen-A. http://imagen-a.us.es/revista/?q=node/31

[10] Gonzalez-Diaz R., Jimenez, M.J., Medrano B., Real P.: Extending the Notion of AT-Model for Intenger Homology Computation. Proc. of the 
2007 International Workshop on Graph-Based Representations in Pattern Recognition, Alicante, Spain, June 11-13, LNCS v. 4538, 330-339 (2007)

[11] Iliopoulos O.S.: Worst-case Complexity Bounds on Algorithms for Computing the Canonical Structure of Finite Abelian Groups and the Hermite and Smith Normal Forms of an Integer Matrix. SIAM J. Comput v. 18, 658-669 (1989)

[12] S. MacLane. Homology, Classics in Mathematics, Springer-Verlag, Berlin, 1995. Reprint of the 1975 edition

[13] Massey W.M.: A Basic Course in Algebraic Topology. Series: Graduate Texts in Mathematics v. 127, 1991

[14] Munkres J.R.: Elements of Algebraic Topology. Addison-Wesley Co., 1984

[15] Peltier S., Alayrangues S., Fuchs L., Lachaud J.: Computation of Homology Groups and Generators. Computers and Graphics v. 30, 62-69 (2006)

[16] Peltier S., Fuchs L., Lienhardt P.: Homology of Simploidal Set. Proc. of the 2006 International Conference on Discrete Geometry for Computer Imagery, Szeged, Hungary, October 25-27, LNCS v. 4245, 235-246 (2006)

[17] Sergeraert F.: Homologie effective. I, II. C. R. Acad. Sci. Paris Sr. I Math. 304, v. 11, 279-282, v. 12, 319-321 (1987)

[18] Storjohann A.: Near Optimal Algorithms for Computing Smith Normal Forms of Integral Matrices. Proc. of the 1996 International Symposium on Symbolic and Algebraic Computation, Zurich, Switzerland, 267-274 (1996)

\section{Appendix: Proof of Theorem 4.14}

Let $A=\left\{a^{1}, \ldots, a^{m}\right\}$ such that if $i<j$ then $a^{i}$ was added to $A$ before $a^{j}$ when the algorithm was applied. Fixed $i$, assume that $f_{r-1} d_{r}\left(a^{k}\right)=d_{r}^{\prime} f_{r}\left(a^{k}\right)$, $a^{k}-g_{r} f_{r}\left(a^{k}\right)=\phi_{r-1} d_{r}\left(a^{k}\right)+d_{r+1} \phi_{r}\left(a^{k}\right), f_{r+1} \phi_{r}\left(a^{k}\right)=0$ and, if $a^{k} \in C_{r}^{\prime}$, $d_{r} g_{r}\left(a^{k}\right)=g_{r-1} d_{r}^{\prime} g_{r}\left(a^{k}\right)$ and $f_{r} g_{r}\left(a^{k}\right)=a^{k}$, for $1 \leq k<i$ and $r=\operatorname{dim}\left(a^{k}\right)$. 
Let $q=\operatorname{dim}\left(a^{i}\right)$. We have to prove that the redefined maps that we will denote here by $d^{\prime \prime}$ (the redefined map of $d^{\prime}$ ), $f^{\prime}, g^{\prime}$ and $\phi^{\prime}$ satisfy that $f_{r-1}^{\prime} d_{r}\left(a^{k}\right)=d_{r}^{\prime \prime} f_{r}^{\prime}\left(a^{k}\right), a^{k}-g_{r}^{\prime} f_{r}^{\prime}\left(a^{k}\right)=\phi_{r-1}^{\prime} d_{r}\left(a^{k}\right)+d_{r+1} \phi_{r}^{\prime}\left(a^{k}\right), f_{r+1}^{\prime} \phi_{r}^{\prime}\left(a^{k}\right)=$ 0 and, if $a^{k} \in C_{r}^{\prime}, d_{r} g_{r}^{\prime}\left(a^{k}\right)=g_{r-1}^{\prime} d_{r}^{\prime \prime} g_{r}^{\prime}\left(a^{k}\right)$ and $f_{r}^{\prime} g_{r}^{\prime}\left(a^{k}\right)=a^{k}$, for $1 \leq k<i$ and $r=\operatorname{dim}\left(a^{k}\right)$.

We have to consider two cases. (1) If $a^{i}$ was added to $A$ while $\min \left\{\mid\left\langle f_{q-1} d_{q}(c)\right.\right.$, $\left.\left.c^{\prime}\right\rangle \mid \neq 0: c \in C_{q}, c^{\prime} \in C_{q-1}^{\prime}\right\}=1$, then $d^{\prime \prime}=d^{\prime}$ and $g^{\prime}=g$ (that is, $d^{\prime}$ and $g$ do not change). Therefore, if $a^{k} \in H_{r}$ for some $r$, then $d_{r} g_{r}^{\prime}\left(a^{k}\right)=d_{r} g_{r}\left(a^{k}\right)=$ $g_{r-1} d_{r}^{\prime}\left(a^{k}\right)=g_{-1}^{\prime} d_{r}^{\prime \prime}\left(a_{k}\right)$ for all $1 \leq k<i$. Consider the element $\alpha \in C_{q-1}^{\prime}$ that defines the auxiliary parameter $x=\left\langle f_{q-1} d_{q}\left(a^{i}\right), \alpha\right\rangle$ at this stage (observe that $x= \pm 1$, then $x x=1$ ).

If $k=i$, then $a^{i} \notin C_{q}^{\prime}$. We have that $f_{q-1}^{\prime} d_{q}\left(a^{i}\right)=f_{q-1} d_{q}\left(a^{i}\right)-x\left\langle f_{q-1} d_{1}\left(a^{i}\right)\right.$, $\alpha\rangle \gamma=\gamma-x x \gamma=0=d_{q}^{\prime \prime} f_{q}^{\prime}\left(a^{i}\right)$ and $\phi_{q-1}^{\prime} d_{q}\left(a^{i}\right)+d_{q+1} \phi_{q}^{\prime}\left(a^{i}\right)=\phi_{q-1}^{\prime} d_{q}\left(a^{i}\right)=$ $\phi_{q-1} d_{q}\left(a^{i}\right)+x\left\langle f_{q-1} d_{q}\left(a^{i}\right), \alpha\right\rangle \gamma^{\prime}=\phi_{q-1} d_{q}\left(a^{i}\right)+x x\left(a^{i}-\phi_{q-1} d_{q}\left(a^{i}\right)\right)=a^{i}$. Moreover, $f_{q+1}^{\prime} \phi_{q}^{\prime}\left(a^{i}\right)=0$ since $\phi_{q}^{\prime}\left(a^{i}\right)=0$.

Observe that: For any $r$-chain $b$ such that $r<q-1, f_{r}^{\prime}(b)=f_{r}(b)$ and $\phi_{r}^{\prime}(b)=\phi_{r}(b) . d_{q}\left(\gamma^{\prime}\right)=d_{q}\left(a^{i}-\phi_{q-1} d_{q}\left(a^{i}\right)\right)=d_{q}\left(a^{i}\right)-\left(d_{q}\left(a^{i}\right)-g_{q-1} f_{q-1} d_{q}\left(a^{i}\right)-\right.$ $\left.\phi_{q-1} d_{q-1} d_{q}\left(a^{i}\right)\right)=g_{q-1} f_{q-1} d_{q}\left(a^{i}\right)=g_{q-1}(\gamma)$. For any $q$-chain $b, f_{q}(b)=0$ and $\phi_{q}(b)=0$, therefore, $f_{q}^{\prime}(b)=0$ and $\phi_{q}^{\prime}(b)=0$.

For $1 \leq k<i$. (1.a) If $r:=\operatorname{dim}\left(a^{k}\right)<q-1$ then $f_{r-1}^{\prime} d_{r}\left(a^{k}\right)=$ $f_{r-1} d_{r}\left(a^{k}\right)=d_{r-1}^{\prime} f_{r}\left(a^{k}\right)=d_{r-1}^{\prime \prime} f_{r}^{\prime}\left(a^{k}\right)$ and $\phi_{r-1}^{\prime} d_{r}\left(a^{k}\right)+d_{r+1} \phi_{r}^{\prime}\left(a^{k}\right)=\phi_{r-1} d_{r}\left(a^{k}\right)+$ $d_{r+1} \phi_{r}\left(a^{k}\right)=a^{k}-g_{r} f_{r}\left(a^{k}\right)=a^{k}-g_{r} f_{r}^{\prime}\left(a^{k}\right)$. Moreover, $f_{r+1}^{\prime} \phi_{r}^{\prime}\left(a^{k}\right)=f_{r+1}^{\prime} \phi_{r}\left(a^{k}\right)=$ $\left.f_{r+1} \phi_{r}\left(a^{k}\right)-x\left\langle f_{r+1} \phi_{r}\left(a^{k}\right), \alpha\right\rangle \gamma\right)=0$ by induction. If $a^{k} \in C_{r}^{\prime}$ then $f_{r}^{\prime} g_{r}\left(a^{k}\right)=$ $f_{r} g_{r}\left(a^{k}\right)=a^{k}$. (1.b) If $\operatorname{dim}\left(a^{k}\right)=q-1$, then $f_{q-2}^{\prime} d_{q-1}\left(a^{k}\right)=f_{q-2} d_{q-1}\left(a^{k}\right)=$ $d_{q-1}^{\prime} f_{q-1}\left(a^{k}\right)=d_{q-1}^{\prime} f_{q-1}\left(a^{k}\right)-x\left\langle f_{q-1}(b), \alpha\right\rangle f_{q-2} d_{q-1} d_{q}(\beta)=d_{q-1}^{\prime} f_{q-1}\left(a^{k}\right)-$ $d_{q-1}^{\prime}\left(x\left\langle f_{q-1}(b), \alpha\right\rangle f_{q-2} d_{q}(\beta)\right)=d_{q-1}^{\prime} f_{q-1}^{\prime}\left(a^{k}\right)=d_{q-1}^{\prime \prime} f_{q-1}^{\prime}\left(a^{k}\right)$ and $\phi_{q-2}^{\prime} d_{q-1}\left(a^{k}\right)+$ $d_{q} \phi_{q-1}^{\prime}\left(a^{k}\right)=\phi_{q-2} d_{q-1}\left(a^{k}\right)+d_{q} \phi_{q-1}\left(a^{k}\right)+x\left\langle f_{q-1}\left(a^{k}\right), \alpha\right\rangle d_{q}\left(\gamma^{\prime}\right)=a^{k}-g_{q-1} f_{q-1}\left(a^{k}\right)+$ $x\left\langle f_{q-1}\left(a^{k}\right), \alpha\right\rangle g_{q-1}(\gamma)=a^{k}-g_{q-1} f_{q-1}^{\prime}\left(a^{k}\right)$. Moreover, $f_{q}^{\prime} \phi_{q-1}^{\prime}\left(a^{k}\right)=f_{q}^{\prime}\left(\phi_{q-1}\left(a^{k}\right)+\right.$ $\left.x\left\langle f_{q-1}\left(a^{k}\right), \alpha\right\rangle \gamma^{\prime}\right)$. Now, $f_{q}^{\prime} \phi_{q-1}\left(a^{k}\right)=0$ since for any $q$-chain $b, f_{q}^{\prime}(b)=0$; $f_{q}^{\prime}\left(\gamma^{\prime}\right)=f_{q}^{\prime}\left(a^{i}-\phi_{q-1} d_{q}\left(a^{i}\right)\right)=0$ for the same reason than above. Then, $f_{q}^{\prime} \phi_{q-1}^{\prime}\left(a^{k}\right)=0$. If $a^{k} \in C_{q-1}^{\prime}$ then $a^{k} \neq \alpha$ and $f_{q-1}^{\prime} g_{q-1}\left(a^{k}\right)=f_{q-1} g_{q-1}\left(a^{k}\right)-$ $x\left\langle f_{q-1} g_{q-1}\left(a^{k}\right), \alpha\right\rangle \gamma=a^{k}-x\left\langle a^{k}, \alpha\right\rangle \gamma=a^{k}$. (1.c) If $\operatorname{dim}\left(a^{k}\right)=q$, then $f_{q-1}^{\prime} d_{q}\left(a^{k}\right)=f_{q-1} d_{q}\left(a^{k}\right)-x\left\langle f_{q-1} d_{q}\left(a^{k}\right), \alpha\right\rangle \gamma=d_{q}^{\prime} f_{q}\left(a^{k}\right)-x\left\langle d_{q}^{\prime} f_{q}\left(a^{k}\right), \alpha\right\rangle \gamma=$ $0=d_{q}^{\prime \prime} f_{q}^{\prime}\left(a^{k}\right)$ and $\phi_{q-1}^{\prime} d_{q}\left(a^{k}\right)+d_{q+1} \phi_{q}^{\prime}\left(a^{k}\right)=\phi_{q-1} d_{q}\left(a^{k}\right)+d_{q+1} \phi_{q}\left(a^{k}\right)+$ $x\left\langle f_{q-1} d_{q}\left(a^{k}\right), \alpha\right\rangle \gamma^{\prime}=a^{k}-g_{q} f_{q}\left(a^{k}\right)+x\left\langle d_{q}^{\prime} f_{q}\left(a^{k}\right), \alpha\right\rangle \gamma^{\prime}=a^{k}-g_{q} f_{q}\left(a^{k}\right)=$ $a^{k}-g_{q} f_{q}^{\prime}\left(a^{k}\right)$. Moreover, $f_{q+1}^{\prime} \phi_{q}^{\prime}\left(a^{k}\right)=0$.

(2) If $a^{i}$ was added to $A$ when $\left\{\left|\left\langle f_{q-1} d_{q}(c), c^{\prime}\right\rangle\right| \neq 0: c \in C_{q}, c^{\prime} \in\right.$ $\left.C_{q-1}^{\prime}\right\}=\emptyset$ or $\min \left\{\left|\left\langle f_{q-1} d_{q}(c), c^{\prime}\right\rangle\right| \neq 0: c \in C_{q}, c^{\prime} \in C_{q-1}^{\prime}\right\}>1$ then, 
$f_{q-1}^{\prime} d_{q}\left(a^{i}\right)=f_{q-1} d_{q}\left(a^{i}\right)=d_{q}^{\prime \prime}\left(a^{i}\right)=d_{q}^{\prime \prime} f_{q}^{\prime}\left(a^{i}\right)$ and $a^{i}-g_{q}^{\prime} f_{q}^{\prime}\left(a^{i}\right)=a^{i}-$ $g_{q}^{\prime}\left(a^{i}\right)=a^{i}-\left(a^{i}-\phi_{q-1} d_{q}\left(a^{i}\right)\right)=\phi_{q-1} d_{q}\left(a^{i}\right)=\phi_{q-1}^{\prime} d_{q}\left(a^{i}\right)+d_{q+1} \phi_{q}^{\prime}\left(a^{i}\right)$. Second, $d_{q} g_{q}^{\prime}\left(a^{i}\right)=d_{q}\left(a^{i}\right)-d_{q} \phi_{q-1} d_{q}\left(a^{i}\right)=g_{q-1} f_{q-1} d_{q}\left(a^{i}\right)+\phi_{q-2} d_{q-1} d_{q}\left(a^{i}\right)=$ $g_{q-1} f_{q-1} d_{q}\left(a^{i}\right)=g_{q-1} d_{q}^{\prime \prime}\left(a^{i}\right)=g_{q-}^{\prime} d_{q}^{\prime \prime}\left(a^{i}\right)$ and $f_{q}^{\prime} g_{q}^{\prime}\left(a^{i}\right)=f_{q}^{\prime}\left(a^{i}-\phi_{q-1} d_{q}\left(a^{i}\right)\right)=$ $a^{i}-f_{q} \phi_{q-1} d_{q}\left(a^{i}\right)=a^{i}$, since $f_{r+1} \phi_{r}\left(a^{k}\right)=0$ for $1 \leq k<i$ and $r=\operatorname{dim}\left(a^{k}\right)$, by induction. Finally, $f_{q+1}^{\prime} \phi_{q}^{\prime}\left(a^{i}\right)=0$ since $\phi_{q}^{\prime}\left(a^{i}\right)=0$. 


\section{STATEMENT}

We present some notes in response to the suggestions made by the reviewers.

Concerning the comments of Reviewer 1, the main remark is that we should add further explanations for the readers who are not specialist in the field. We have followed this philosophy. We could classify the suggestions in the following categories:

- Little gaps or changes of notations: we have followed all the reviewer's instructions.

- Addition of examples: we have added more examples to illustrate some aspects that the reviewer asked to be clarified in this way.

- Further explanations: we have recalled more background concepts and detailed some technical aspects at the points that the referee suggested. In some other cases, we have simply extended the given explanations.

- Regarding Algorithm 1 (Algorithm 13 in the previous version), it has been modified since it was difficult to follow the technical aspects, as pointed out by the reviewer. The changes have allowed to carry out the proof of Theorem 16 (Theorem 14 in the previous version)in a clearer way.

- Proof of Theorem 19 (Theorem 18 in the previous version) has been provided in an appendix.

- With respect to the reviewer's question about the reduction of the complexity using Alg. 2 (Alg. 17 in the previous version), we have to say that it is difficult to quantify such as a reduction in general. We

plan to study this question in detail and expect to provide experiments and concrete examples of such a reduction.

As for the comments of Reviewer 2, all the suggestions have been carried out. Most of them are Grammar errors or little gaps that have been corrected. Also the proposed change in the numbering for definitions, theorems, etc., has been undertaken. 


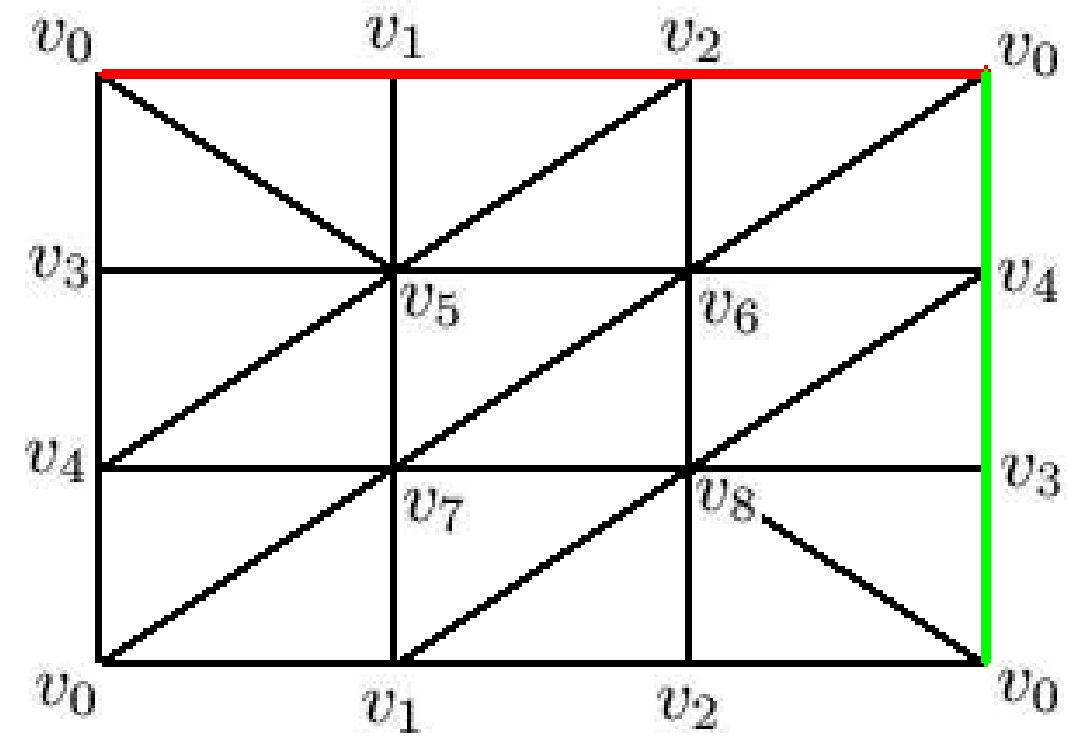





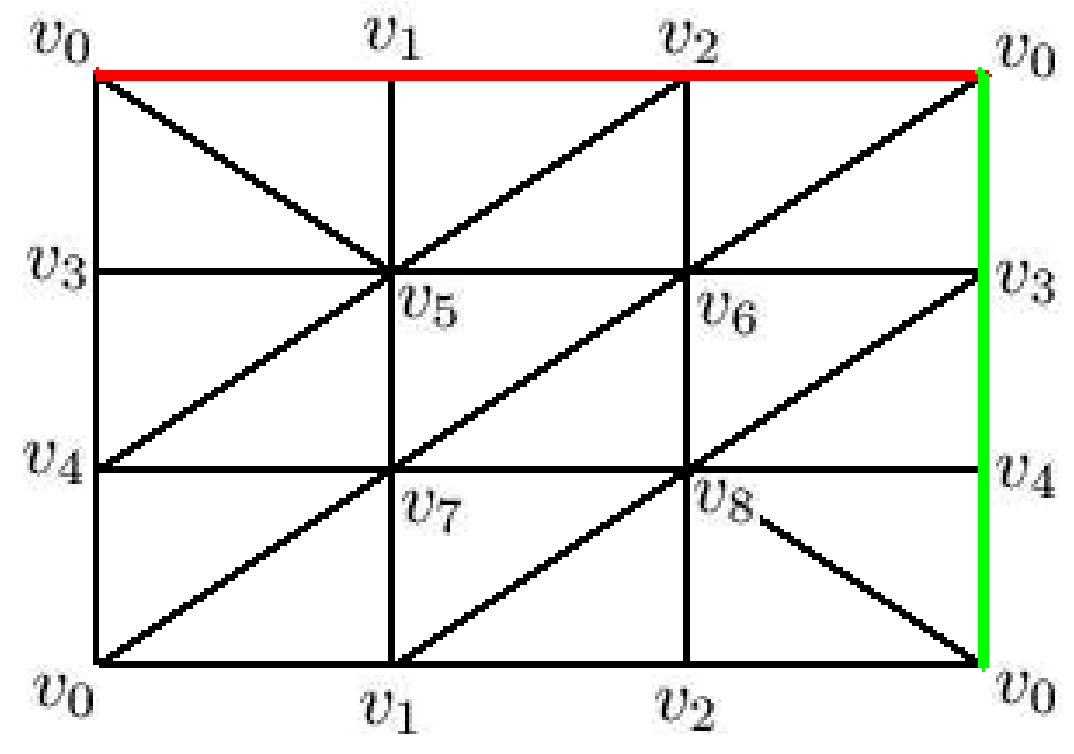




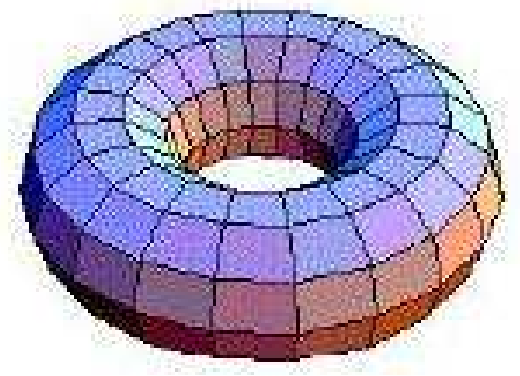

